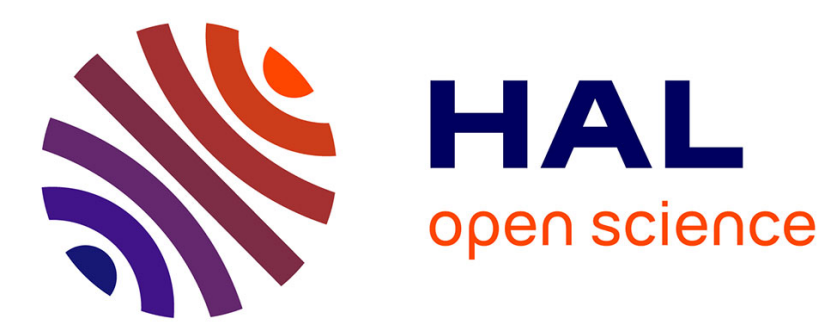

\title{
On the standing wave problem in deep water \\ Gérard Iooss
}

\section{To cite this version:}

Gérard Iooss. On the standing wave problem in deep water. Journal of Mathematical Fluid Mechanics, 2002, 4, pp.31. hal-01271053

\section{HAL Id: hal-01271053 \\ https://hal.univ-cotedazur.fr/hal-01271053}

Submitted on 9 Feb 2016

HAL is a multi-disciplinary open access archive for the deposit and dissemination of scientific research documents, whether they are published or not. The documents may come from teaching and research institutions in France or abroad, or from public or private research centers.
L'archive ouverte pluridisciplinaire HAL, est destinée au dépôt et à la diffusion de documents scientifiques de niveau recherche, publiés ou non, émanant des établissements d'enseignement et de recherche français ou étrangers, des laboratoires publics ou privés. 


\title{
On the standing wave problem in deep water
}

\author{
Gérard Iooss \\ Institut Universitaire de France \\ INLN UMR CNRS-UNSA 6618 \\ 1361 route des Lucioles, F-06560 Valbonne \\ e-mail: iooss@inln.cnrs.fr
}

\begin{abstract}
We present a new formulation of the classical two-dimensional standing wave problem which makes transparent the (seemingly mysterious) elimination of the quadratic terms made in [6]. Despite the presence of infinitely many resonances, corresponding to an infinite dimensional kernel of the linearized operator, we solve the infinite dimensional bifurcation equation by uncoupling the critical modes up to cubic order, via a Lyapunov-Schmidt like process. This is done without using a normalization of the cubic order terms as in [6], where the computation contains a mistake, although the conclusion was in the end correct. Then we give all possible bifurcating formal solutions, as powers series of the amplitude (as in [6]), with an arbitrary number, possibly infinite, of dominant modes.
\end{abstract}

\section{Introduction}

The two-dimensional standing wave problem for a potential flow with a free surface has attracted lot of interest since Stokes, and specially very recently. In particular the existence question in the cae of finite depth has a solution thanks to the work of Plotnikov and Toland [7]. They use, in an essential way, the fact that for most of the values of the depth, the kernel of the linearized operator is one dimensional. The complication there comes from a small divisor problem in the control of the norm of the inverse of the linearized operator near the solution (because, as seems unavoidable, they use the Nash Moser theorem). In the present paper we consider the infinite depth case and we do not prove the existence of a solution (still an open problem), but instead we give a new formulation of the problem, and use it to show the existence of formal solutions in the form of power series in the amplitude, with infinitely many possibilities for the choice of the dominant modes. This solves the algebraic problem of the infinitely many resonances, which are not present in the finite depth case. This result was also obtained in our previous work [6], where the formulation used is 
non so transparent. The presentation here is drastically different from [6]. We propose a new formulation in terms of analytic functions instead of Fourier series. In addition a mistake (very hidden), which occured in the computation of resonant cubic terms in [6], is corrected below. The results are (miraculously) the same as in [6].

Let us notice in addition that we do not use here a hamiltonian formulation, as it was introduced by Zakharov [9], and which was used in particular in [5], [3], [4]. These authors consider the evolutionary problem for spatially periodic solutions, and consider the normal form of the hamiltonian infinite dimentional vector field. The absence of resonant terms at quadratic order of the vector field is shown, and the resonant cubic terms in the vector field are computed by Craig and Worfolk in [3], showing that the system is integrable if truncated at cubic order, as was announced by Dyachenko and Zakharov in [5], and not integrable if considered at higher order. In the present work, we give explicitely the complete form of the system without quadratic terms, obtained after a simple change of variables not needing a hamiltonian formulation. Moreover, the infinite set of bifurcation equations, leading to the infinite set of formal solutions for the standing wave problem, is obtained with no need of killing the non resonant terms by a new change of variables. In principle the principal part of this set of equations might be obtained directly with the work done in [3], since these authors have all possible solutions of the system truncated at cubic order, however we prefer to stay at a more elementary and explicit level, for preparing the work for a future proof of the existence of all these standing waves.

Let us now explain in detail our result. We denote by $y<h(x, t)$ the region occupied by the liquid, where $h$ is the height of the free surface and we look for time periodic (period $T$ ), and $x$-periodic flows (wave length $\lambda$ ). Choosing respectively $T / 2 \pi, \lambda / 2 \pi, \lambda / T, \lambda^{2} / 2 \pi T$ as scales of time, length, velocity and potential, we obtain the dimensionless system of equations for the potential $\phi(x, y, t)$ and height $h$ :

$$
\left.\begin{array}{rl}
\Delta \phi=0, \quad \text { in }-\infty & <y<h(x, t), \\
\frac{\partial h}{\partial t}+u \frac{\partial h}{\partial x}-v & =0 \\
\frac{\partial \phi}{\partial t}+\frac{1}{2}\left(u^{2}+v^{2}\right)+(1+\mu) h & =0
\end{array}\right\} \text { on } y=h(x, t),
$$

where the velocity components $(u, v)$ satisfy $u=\partial \phi / \partial x, v=\partial \phi / \partial y$, and where $1+\mu=g T^{2} / 2 \pi \lambda, g$ being the acceleration due to gravity. We denote by $f(z, t)=\phi(x, y, t)+i \psi(x, y, t)$ the complex potential, analytic in the domain $\operatorname{Im} z<h(x, t)$, where $z=x+i y$, and $\psi$ is the stream function. In the following we are interested in solutions such that

$$
\begin{aligned}
& f(z+2 \pi, t)=f(z, t+2 \pi)=f(z, t), \\
& h(x+2 \pi, t)=h(x, t+2 \pi)=h(x, t),
\end{aligned}
$$


with evenness properties in $t$

$$
\begin{aligned}
& f(z,-t)=-f(z, t), \\
& h(x,-t)=h(x, t),
\end{aligned}
$$

and invariant by vertical miror symmetry (as in [1])

$$
\begin{aligned}
f(-\bar{z}, t) & =\bar{f}(z, t), \\
h(-x, t) & =h(x, t) .
\end{aligned}
$$

This analyticity imposes a special form of the Fourier expansions in $x$, so that we can write

$$
\begin{aligned}
f(z, t) & =\sum_{p \geq 0} f_{p}(t) e^{-i p z}, \\
\phi(x, y, t) & =\sum_{p \geq 0} \operatorname{Re}\left(f_{p}(t) e^{-i p x}\right) e^{p y}, \\
h(x, t) & =\sum_{p \geq 0} \operatorname{Re}\left(h_{p}(t) e^{-i p x}\right) .
\end{aligned}
$$

The linearized system of (1), (2) near 0 (a flat free surface) with boundary conditions $(4,5)$ gives solutions of the form

$$
\begin{aligned}
h(x, t) & =\cos q t \cos p x, \\
\phi(x, y, t) & =-\frac{q}{p} e^{p y} \sin q t \cos p x,
\end{aligned}
$$

provided that $(1+\mu) p=q^{2}$. This gives non zero solutions whenever $(1+\mu)$ is a positive rational number $r / s$. Then $q=k r, p=k^{2} r s, k=1,2, \ldots$ give infinitely many linearly independent solutions for the same values of $\mu$. We shall consider the case where $(1+\mu)$ is near 1 , since all other cases reduce to this case after a suitable rescaling: dividing the scale of time by $r$, and the length scale by $r s$, multiplies $(1+\mu)$ by $s / r$.

The fact that at any rational $\mu$, there is an infinite dimensional set of solutions creates big difficulties, known as "infinitely many resonances". Indeed, for any solution of the problem, the nonlinear terms of the system have to satisfy infinitely many conditions!

In the paper [1], Amick \& Toland justified the algorithmic approach conjectured by Schwartz \& Whitney [8]. Looking for solutions symmetric under reflexion $x \rightarrow-x$ and even in time $t$, they prove that if one chooses the dominant mode as $h(x, t)=\varepsilon \cos t \cos x$, the resonances do not arise at any stage of the computation of the expansion in powers of $\varepsilon$, where $\varepsilon=2 \sqrt{\mu}$. In this approach, the system is expressed in the form of an infinite system of coupled ordinary differential equations in the time-periodic spatial Fourier series components of the standing wave. 
In what follows, we start with the formulation of [8] and [1], and reformulate the problem in terms of analytic functions on the complex half plane. In addition to the resonance problem mentioned above, the occurence of quadratic terms in [1] is the source of major difficulties, due to the couping of each mode with infinitely many other modes, and a direct application of a Lyapunov-Schmidt like method does not seem tractable. We derive below a new formulation without quadratic terms, using a suitable choice of variables and coordinates. This formulation explains the mysterious change of variables made in [6] and has the advantage to be hopefully useful for further analysis on the existence of standing waves (beyond what is done below).

With such a formulation, we are able to solve the infinitely - many resonances problem, using, as in [6] a Lyapunov-Schmidt type of method for proving that one can construct the expansion in powers of $\varepsilon=2 \sqrt{\mu}$, in taking a dominant part of the height $h(x, t)$ of the form

$$
h(x, t) \sim \varepsilon \sum_{q \in I} \frac{( \pm 1)_{q}}{q^{2}} \cos q^{2} x \cos q t
$$

where the number of basic modes given by $I \subset \mathbb{N}$ may be infinite, and $( \pm 1)_{q}= \pm 1$. This general result is in accordance with the numerical results of Bryant and Stiassnie [2] who considered the cases $I=\{1,2\}$ and $I=$ $\{1,2,3\}$.

An important feature of our new approach is that we do not need to put the cubic terms into normal form (contrary to the claim in [6], where, in addition, a mistake was made in the calculation of the normal form, here corrected in Appendix B, where the agebraic problem is completely solved). Indeed, it appears that such a normal form contains too many terms to be useful. A general Lyapunov-Schmidt technique is more flexible and better adapted for dealing with periodic problems.

Finally, it should be noted that our result is only valid for the formal expansions of these various "solutions" in powers of $\varepsilon=2 \sqrt{\mu}$, the convergence in some function space is still an open problem.

\section{Notations}

Let us denote by

$$
\mathbb{C}_{\delta}=\mathbb{R} \times(-\infty, \delta), \delta \geq 0
$$

a domain in the complex plane $\mathbb{C}$ shifted from the standard complex half plane (denoted here by $\mathbb{C}_{0}$ ). Now, for any continuous function $f: \mathbb{R} \rightarrow \mathbb{C}$, $2 \pi$ - periodic in $\xi$, define the analytic function $\mathcal{T} f$, for $\zeta \in \mathbb{C}_{0}$, by

$$
\mathcal{T} f(\zeta)=\frac{1}{2 \pi} \int_{0}^{2 \pi} \frac{f(\theta)}{e^{i(\zeta-\theta)}-1} d \theta
$$

Then we have the following elementary properties. 
Lemma 1 For any Lipschitz continuous function $f: \mathbb{R} \rightarrow \mathbb{C}, 2 \pi-$ periodic in $\xi$, denote its Fourier series by

$$
f(\xi)=\sum_{p \in \mathbb{Z}} f_{p} e^{-i p \xi} .
$$

(a)

$$
\mathcal{T} f(\zeta)=\sum_{p \geq 1} f_{p} e^{-i p \zeta},
$$

holds, where $\zeta=\xi+i \eta \in \mathbb{C}_{0}$.

(b) If $f$ is real-valued, then

$$
f(\xi)=[f]+\left.2 \operatorname{Re} \mathcal{T} f\right|_{\eta=0},
$$

where $[f]$ denotes the average of $f$ over one period. If $f$ is imaginary valued, then

$$
f(\xi)=[f]+\left.2 i \operatorname{Im} \mathcal{T} f\right|_{\eta=0} .
$$

(c) If $f$ is analytic on $\mathbb{R}$ with a radius of convergence $\delta$, then $\mathcal{T} f(\zeta)$ has an analytic continuation, also denoted by $\mathcal{T} f(\zeta)$, to $\mathbb{C}_{\delta}$.

(d) If $f$ has an analytic continuation in $\mathbb{C}_{0}$, then

$$
\begin{aligned}
& \mathcal{T} f(\zeta)=f(\zeta)-[f], \\
& \mathcal{T} \bar{f}(\zeta)=0 .
\end{aligned}
$$

About the point (c) notice that $\mathcal{T}$, as defined by the integral formula, is zero on $\mathbb{C}_{\delta} \backslash\left\{\right.$ closure of $\left.\mathbb{C}_{0}\right\}$ (by Cauchy's integral formula). Another remark is that the periodic Hilbert transform $\mathcal{C}$ is defined by

$$
\begin{aligned}
\mathcal{C}\left(e^{i p \xi}\right) & =-i \operatorname{sgn}(p) e^{i p \xi}, \quad p \in \mathbb{Z} \backslash\{0\}, \\
& =0 \text { for } p=0,
\end{aligned}
$$

and, for any Lipschitz continuous function $f: \mathbb{R} \rightarrow \mathbb{C}, 2 \pi$ - periodic in $\xi$ we have the identity

$$
f-i \mathcal{C} f=[f]+\left.2 \mathcal{T} f\right|_{\eta=0},
$$

and when $f$ is real-valued, the function $f-i \mathcal{C} f$ may be extended analytically in $\mathbb{C}_{0}$, where our $\mathcal{T} f(\zeta)$ gives the extension.

\section{Formulation of the problem}

We use a variation of the formulation used in [1] and [8]. Let us introduce a time dependent conformal injection in $\mathbb{C}_{\delta}$, for each $t \in \mathbb{R}$, defined by $\mathbb{I}+\widetilde{Z}(\cdot, t)$ where $\mathbb{I}(\zeta)=\zeta, \zeta=\xi+i \eta$,

$$
\widetilde{Z}(\zeta+2 \pi, t)=\widetilde{Z}(\zeta, t+2 \pi)=\widetilde{Z}(\zeta, t),
$$


and define, for $\|\widetilde{Z}\|_{\infty}$ sufficiently small,

$$
\begin{aligned}
S_{\widetilde{Z}} & =\left\{(\xi+\widetilde{Z}(\xi, t), t) ;(\xi, t) \in \mathbb{R}^{2}\right\}, \quad \text { (free boundary) } \\
\Omega_{\widetilde{Z}} & =\left\{(\zeta+\widetilde{Z}(\zeta, t), t) ; \quad \zeta \in \mathbb{C}_{0}, t \in \mathbb{R}\right\} \subset \mathbb{C}_{\delta} \times \mathbb{R}, \\
\Omega_{\widetilde{Z}_{t}} & =\left\{z ;(z, t) \in \Omega_{\widetilde{Z}}\right\} \subset \mathbb{C}_{\delta} .
\end{aligned}
$$

The physical domain of the flow is then given by $z=x+i y \in \Omega_{\widetilde{Z}_{t}}$, which may be transformed into $\mathbb{C}_{0}$ by the inverse mapping (we omit $t$ in the writing of the mappings)

$$
(\mathbb{I}+\widetilde{Z})^{-1} \stackrel{\text { def }}{=} \mathbb{I}-Z: \Omega_{\widetilde{Z}_{t}} \rightarrow \mathbb{C}_{0}
$$

We denote the complex potential by $F(\zeta, t)(=f(z, t))$, and the complex velocity by

$$
u-i v=f_{z}^{\prime}(z, t)=W(z, t),
$$

and the miror-symmetry condition (5) leads to

$$
\begin{aligned}
W(-\bar{z}, t) & =-\bar{W}(z, t), \\
F(-\bar{\zeta}, t) & =\bar{F}(\zeta, t), \\
\widetilde{Z}(-\bar{\zeta}, t) & =-\overline{\widetilde{Z}}(\zeta, t), \\
Z(-\bar{z}, t) & =-\bar{Z}(z, t) .
\end{aligned}
$$

We also need the following notation for $(\xi, t) \in \mathbb{R}^{2}$

$$
\begin{gathered}
\widetilde{D}(\xi, t)=\widetilde{Z}_{t}^{\prime}(\xi, t) \overline{\widetilde{Z}}_{\xi}^{\prime}(\xi, t)-\overline{\widetilde{Z}}_{t}^{\prime}(\xi, t) \widetilde{Z}_{\xi}^{\prime}(\xi, t), \\
\widetilde{C}(\xi, t)=\left|f_{z}^{\prime}(\xi+\widetilde{Z}(\xi, t), t)\right|^{2} .
\end{gathered}
$$

In this section we show the following

Lemma 2 With the above choice of variables, the system (1,2) for miror symmetric solutions, may be transformed into the following system

$$
\begin{gathered}
f_{z}^{\prime}+Z_{t}^{\prime}+\left(1-Z_{z}^{\prime}\right)\left\{\mathcal{T} \widetilde{D}+\frac{1}{2}[\widetilde{D}]\right\} \circ(\mathbb{I}-Z)=0, \\
f_{t}^{\prime}-i(1+\mu) Z+\left\{\mathcal{T} \widetilde{C}+\frac{1}{2}[\widetilde{C}]\right\} \circ(\mathbb{I}-Z)=0,
\end{gathered}
$$

for $(z, t) \in \Omega_{\widetilde{Z}}$.

Proof: With our choice of coordinates and variables the kinematic boundary condition $(2)_{1}$ on $S_{\widetilde{Z}}$ takes the following form (see [8],[1] for the first equation)

$$
\operatorname{Im}\left\{\left(1+\widetilde{Z}_{\xi}^{\prime}(\xi, t)\right) W(\xi+\widetilde{Z}(\xi, t), t)+\widetilde{Z}_{t}^{\prime}(\xi, t)\left(1+\widetilde{Z}_{\xi}^{\prime}(\xi, t)\right)\right\}=0,
$$


while the dynamic boundary condition $(2)_{2}$, also on $S_{\widetilde{Z}}$, takes the form

$$
\operatorname{Re}\left\{f_{t}^{\prime}(\xi+\widetilde{Z}(\xi, t), t)-i(1+\mu) \widetilde{Z}(\xi, t)+\frac{1}{2}|W(\xi+\widetilde{Z}(\xi, t), t)|^{2}\right\}=0 .
$$

We then modify both these equations, by introducing our operator $\mathcal{T}$ with the aim of rewriting the problem as a system of two analytic equations in the half plane $\mathbb{C}_{0}$. First we have, from (12) and lemma 1,

$$
\begin{aligned}
0 & =\operatorname{Im}\left\{\left(1+\widetilde{Z}_{\xi}^{\prime}(\xi, t)\right) W(\xi+\widetilde{Z}(\xi, t), t)+\widetilde{Z}_{t}^{\prime}(\xi, t)+\right. \\
& \left.+\frac{1}{2}\left[\widetilde{Z}_{t}^{\prime}(\xi, t) \widetilde{\widetilde{Z}}_{\xi}^{\prime}(\xi, t)-\widetilde{\widetilde{Z}}_{t}^{\prime}(\xi, t) \widetilde{Z}_{\xi}^{\prime}(\xi, t)\right]\right\},
\end{aligned}
$$

i.e.

$$
\begin{aligned}
0 & =\operatorname{Im}\left\{\left(1+\widetilde{Z}_{\xi}^{\prime}(\xi, t)\right) W(\xi+\widetilde{Z}(\xi, t), t)+\widetilde{Z}_{t}^{\prime}(\xi, t)\right\}+ \\
& +\operatorname{Im}(\mathcal{T} \widetilde{D})(\xi, t)+\operatorname{Im} \frac{1}{2}[\widetilde{D}](t),
\end{aligned}
$$

and, since we have the imaginary part of a holomorphic $2 \pi$ - periodic function which is 0 on the real line $\eta=0$, we deduce that

$$
\begin{aligned}
c(t) & =\left(1+\widetilde{Z}_{\zeta}^{\prime}\right) W(\zeta+\widetilde{Z}(\zeta, t), t)+\widetilde{Z}_{t}^{\prime}(\zeta, t)+ \\
& +(\mathcal{T} \widetilde{D})(\zeta, t)+i \operatorname{Im} \frac{1}{2}[\widetilde{D}](t),
\end{aligned}
$$

where $c(t)$ is real. From the miror-symmetry conditions (7), it follows that the average quantities $\left[\widetilde{Z}_{t}^{\prime}(\zeta, t)\right]$ and $[\widetilde{D}]$ are imaginary. Now the average of any $\mathcal{T} f$ is 0 , and the average of

$$
\left(1+\widetilde{Z}_{\zeta}^{\prime}\right) W(\zeta+\widetilde{Z}(\zeta, t), t)=F_{\zeta}^{\prime}(\zeta, t)
$$

is 0 by construction, since the velocity $W$ has zero average (due to the periodicity of $f$ in $x$ ). With this choice, there results that

$$
\begin{aligned}
0 & =\left(1+\widetilde{Z}_{\zeta}^{\prime}\right) W(\zeta+\widetilde{Z}(\zeta, t), t)+\widetilde{Z}_{t}^{\prime}(\zeta, t)+ \\
& +(\mathcal{T} \widetilde{D})(\zeta, t)+\frac{1}{2}[\widetilde{D}](t),
\end{aligned}
$$

holds for all $(\zeta, t) \in \mathbb{C}_{0} \times \mathbb{R}$ and, if we now use the coordinates $(z, t)=$ $(\zeta+\widetilde{Z}(\zeta, t), t)$ where

$$
\zeta=z-Z(z, t)
$$

and observe that

$$
\begin{aligned}
1-Z_{z}^{\prime} & =\left(1+\widetilde{Z}_{\zeta}^{\prime}\right)^{-1}, \\
\frac{\widetilde{Z}_{t}^{\prime}(\zeta, t)}{1+\widetilde{Z}_{\zeta}^{\prime}} & =Z_{t}^{\prime}(z, t),
\end{aligned}
$$


we obtain the equation

$$
0=W(z, t)+Z_{t}^{\prime}(z, t)+\left(1-Z_{z}^{\prime}(z, t)\right)\left\{\mathcal{T} \widetilde{D}+\frac{1}{2}[\widetilde{D}(\cdot, t)]\right\}(z-Z(z, t), t)
$$

valid for $(z, t) \in \Omega_{\widetilde{Z}}$. This may be written as (10).

In the same way, introducing the operator $\mathcal{T}$ into (13), leads to

$$
\begin{aligned}
0 & =\operatorname{Re}\left\{f_{t}^{\prime}(\xi+\widetilde{Z}(\xi, t), t)-i(1+\mu) \widetilde{Z}(\xi, t)\right\}+ \\
& +\operatorname{Re} \mathcal{T} \widetilde{C}(\xi, t)+\frac{1}{2}[\widetilde{C}](t) .
\end{aligned}
$$

Since we have the real part of an holomorphic $2 \pi$ - periodic function which is zero on the real line $\eta=0$, this leads to

$$
\begin{aligned}
c_{1}(t) & =f_{t}^{\prime}(\zeta+\widetilde{Z}(\zeta, t), t)-i(1+\mu) \widetilde{Z}(\zeta, t)+ \\
& +(\mathcal{T} \widetilde{C})(\zeta, t)+\frac{1}{2}[\widetilde{C}](t),
\end{aligned}
$$

for $\zeta \in \mathbb{C}_{0}$ and where $c_{1}(t)$ is purely imaginary. If $f$ is replaced by

$$
f(z, t)-\int_{0}^{t} c_{1}(\tau) d \tau
$$

then equations (1),((2) are not affected because $c_{1}$ is imaginary. Moreover, as seen previously, due to the symmetry of the flow, the average $i[\widetilde{Z}]$ is real, like $[\widetilde{C}]$, hence we take $c_{1}(t) \equiv 0$. Then the above equation becomes

$$
f_{t}^{\prime}(z, t)-i(1+\mu) Z(z, t)+(\mathcal{T} \widetilde{C}(\cdot, t))(z-Z(z, t), t)+\frac{1}{2}[\widetilde{C}(\cdot, t)]=0,
$$

valid for $(z, t) \in \Omega_{\widetilde{Z}}$ and which may be written as (11). Notice that we can also write the above equation, after differentiating with respect to $z$, as

$$
W_{t}^{\prime}-i(1+\mu) Z_{z}^{\prime}+\{(\mathcal{T} \widetilde{C}) \circ(\mathbb{I}-Z)\}_{z}^{\prime}=0 .
$$

The system of two equations (10) (11) is the system on which we work in the following sections. The advantage of this system, with respect to the one used in [1] is that we have already eliminated the "pure" analytic terms (i.e. those which do not involve $\mathcal{T}$ ), which gave all the problems appearing under the name of the "Schwartz-Whitney conjecture". This leads to an understanding of why the change of variable made in [6] is able to eliminate the pure analytic quadratic terms in the formulation of $[8],[1]$ (see the acknowledgments). 


\section{Elimination of the remnent quadratic terms}

The usual way for obtaining the bifurcating solutions in the search for periodic solutions is to use the Lyapunov-Schmidt method (see section 8). The presence of quadratic terms in the system (10) (11) implies that the terms cubic in the critical modes (lying in the infinite dimensional kernel of the linearized operator) are very difficult to compute explicitely in the bifurcation equation (infinite dimensional). We shall see that there is no term quadratic in the critical modes in this bifurcation equation (see a remark at section 8), hence the calculation of cubic order is really necessary. So, it is fortunate that we already eliminated many quadratic terms thanks to our new formulation. This is however not sufficient, and, as shown in [6], we can eliminate all quadratic terms via a suitable near-identity polynomial change of variable. We show the same property below, on our formulation of the system (10) (11).

First, we show the following

Lemma 3 Considering functions analytic in $\mathbb{C}_{\delta}$, the change of variables $(Z, f) \mapsto(U, G)$ defined implicitely near 0, by

$$
\begin{aligned}
& Z=U+\mathcal{T}\left(\bar{Z} Z_{x}^{\prime}\right)+\frac{1}{2}\left[\bar{Z} Z_{x}^{\prime}\right], \\
& f=G+\mathcal{T}\left(\bar{Z} f_{x}^{\prime}\right)+\frac{1}{2}\left[\bar{Z} f_{x}^{\prime}\right],
\end{aligned}
$$

transforms the system (10) (11) into

$$
\begin{gathered}
G_{z}^{\prime}+U_{t}^{\prime}+O(3)=0, \\
G_{t}^{\prime}-i(1+\mu) U+O(3)=0,
\end{gathered}
$$

where $O(3)$ means terms at least cubic in $(G, U)$ (we will be precise about the cubic terms later).

This result is the analogue of the one found in using Fourier series in [6] at the beginning of section 3 (where the lengthy computations were hidden to the reader!)

Proof: Let us first identify the quadratic terms in equations (10) (11):

$$
\begin{aligned}
\left(1-Z_{z}^{\prime}\right)\left\{\mathcal{T} \widetilde{D}+\frac{1}{2}[\widetilde{D}]\right\} \circ(\mathbb{I}-Z) & =(\mathcal{T} \widetilde{D})+\frac{1}{2}[\widetilde{D}]+O(3) \\
& =\mathcal{T}\left\{Z_{t}^{\prime} \bar{Z}_{x}^{\prime}-\bar{Z}_{t}^{\prime} Z_{x}^{\prime}\right\}+ \\
& +\frac{1}{2}\left[Z_{t}^{\prime} \bar{Z}_{x}^{\prime}-\bar{Z}_{t}^{\prime} Z_{x}^{\prime}\right]+O(3)
\end{aligned}
$$




$$
\begin{aligned}
\left\{\mathcal{T} \widetilde{C}+\frac{1}{2}[\widetilde{C}]\right\} \circ(\mathbb{I}-Z) & =\mathcal{T} \widetilde{C}+\frac{1}{2}[\widetilde{C}]+O(3) \\
& =\mathcal{T}\left\{\left|f_{z}^{\prime}\right|^{2}\right\}+\frac{1}{2}\left[\left|f_{z}^{\prime}(x, t)\right|^{2}\right]+O(3) .
\end{aligned}
$$

Let us make the change of variables (15). The quadratic terms in the new equation for (10) is

$$
\begin{aligned}
& \left(\mathcal{T}\left(\bar{Z} f_{x}^{\prime}\right)\right)_{z}^{\prime}+\left(\mathcal{T}\left(\bar{Z} Z_{x}^{\prime}\right)\right)_{t}^{\prime}+\mathcal{T}\left\{Z_{t}^{\prime} \bar{Z}_{x}^{\prime}-\bar{Z}_{t}^{\prime} Z_{x}^{\prime}\right\}+ \\
& +\frac{1}{2}\left[\bar{Z} Z_{x}^{\prime}\right]_{t}^{\prime}+\frac{1}{2}\left[Z_{t}^{\prime} \bar{Z}_{x}^{\prime}-\bar{Z}_{t}^{\prime} Z_{x}^{\prime}\right]
\end{aligned}
$$

which may be also written as

$$
\mathcal{T}\left(\left(\bar{Z}\left(f_{z}^{\prime}+Z_{t}^{\prime}\right)\right)_{x}^{\prime}\right)+\frac{1}{2}\left[\left(\bar{Z} Z_{t}^{\prime}\right)_{x}^{\prime}\right]=\left(\mathcal{T}\left(\bar{Z}\left(f_{z}^{\prime}+Z_{t}^{\prime}\right)\right)\right)_{z}^{\prime},
$$

which we notice is of cubic order, since $f_{z}^{\prime}+Z_{t}^{\prime}=O(2)$.

The quadratic terms in the new equation for (11) is

$$
\begin{aligned}
& \mathcal{T}\left(\bar{Z} f_{x}^{\prime}\right)_{t}^{\prime}-i(1+\mu) \mathcal{T}\left(\bar{Z} Z_{x}^{\prime}\right)+\mathcal{T}\left\{\left|f_{z}^{\prime}\right|^{2}\right\}+ \\
& +\frac{1}{2}\left[\bar{Z} f_{x}^{\prime}\right]_{t}^{\prime}-i(1+\mu) \frac{1}{2}\left[\bar{Z} Z_{x}^{\prime}\right]+\frac{1}{2}\left[\left|f_{z}^{\prime}\right|^{2}\right]
\end{aligned}
$$

which may be also written as

$$
\begin{aligned}
& \mathcal{T}\left(\bar{Z}\left(f_{t}^{\prime}-i(1+\mu) Z\right)_{z}^{\prime}\right)+\mathcal{T}\left(f_{z}^{\prime} \overline{\left(f_{z}^{\prime}+Z_{t}^{\prime}\right)}\right)+ \\
& +\frac{1}{2}\left[\bar{Z}\left(f_{t}^{\prime}-i(1+\mu) Z\right)_{z}^{\prime}\right]+\frac{1}{2}\left[f_{z}^{\prime} \overline{\left(f_{z}^{\prime}+Z_{t}^{\prime}\right)}\right]
\end{aligned}
$$

which again is of cubic order, since $f_{z}^{\prime}+Z_{t}^{\prime}=O(2)$ and $f_{t}^{\prime}-i(1+\mu) Z=O(2)$. As a consequence, the change of variables (15) suppresses all quadratic terms in system (10),(11).

\section{Computation of cubic terms of the new system}

We now need to compute precisely at least the cubic terms of the new system, because these cubic terms are crucial for the bifurcation analysis. First, we give some elementary properties of the operator $\mathcal{T}$, which are useful in the sequel, and which complement those given at lemma 1:

Lemma 4 For any function $f: \mathbb{C} \rightarrow \mathbb{C}, 2 \pi-$ periodic in $x$, analytic in $\mathbb{C}_{0}$, Lipschitz continuous on $\mathbb{R} \times\{0\}$, and any function $g: \mathbb{R} \rightarrow \mathbb{C}, 2 \pi-$ periodic in $x$ and Lipschitz continous on $\mathbb{R}$, we have the following identities:

$$
\begin{aligned}
& \text { (a) } \mathcal{T}(f \mathcal{T} g)=f \mathcal{T} g, \\
& \text { (b) } \mathcal{T}(\bar{f} \mathcal{T} g)=\mathcal{T}(\bar{f} g), \\
& \text { (c) } \mathcal{T}(f \overline{\mathcal{T} g})=\mathcal{T}(f \bar{g})-f \mathcal{T}(\bar{g})-f[\bar{g}] .
\end{aligned}
$$


Proof: The two first identities are left to the reader. Let us show the last one.

We first define the Fourier series

$$
\begin{aligned}
& f(z)=\sum_{p \geq 0} f_{p} e^{-i p z}, \\
& g(x)=\sum_{q \in \mathbb{Z}} g_{q} e^{-i q x},
\end{aligned}
$$

then we have successively

$$
\begin{aligned}
f \overline{\mathcal{T} g}(x) & =\sum_{q \geq 1, p \geq 0} f_{p} \bar{g}_{q} e^{-i(p-q) x}, \\
f \mathcal{T} \bar{g}(x) & =\sum_{q \leq-1, p \geq 0} f_{p} \bar{g}_{q} e^{-i(p-q) x}, \\
\mathcal{T} f \bar{g}(x) & =\sum_{p \geq q+1} f_{p} \bar{g}_{q} e^{-i(p-q) x}, \\
\mathcal{T}(f \overline{\mathcal{T} g})(x) & =\sum_{q \geq 1, p \geq q+1} f_{p} \bar{g}_{q} e^{-i(p-q) x},
\end{aligned}
$$

from which the lemma follows easily.

In the next lemma we show how a change of variable $(Z, f) \mapsto(U, G)$, different from, but equivalent at quadratic order to, the one in lemma 3 , simplifies the system $(10,11)$ and facilitates explicit calculation of terms in a Taylor series expansion of the resulting equations. Another advantage of this change of variable is that it is a priori defined for functions analytic in the domain bounded by the free boundary $S_{\widetilde{Z}}$.

Lemma 5 The change of variables $(Z, f) \mapsto(U, G)$ given by

$$
\begin{aligned}
& Z=U+\left(\mathcal{T}\left(\overline{\widetilde{Z}} \widetilde{Z}_{\xi}^{\prime}\right)\right) \circ(\mathbb{I}-Z)+\frac{1}{2}\left[\overline{\widetilde{Z}} \widetilde{Z}_{\xi}^{\prime}\right], \\
& f=G+\left(\mathcal{T}\left(\overline{\widetilde{Z}} f_{z}^{\prime}(\cdot+\widetilde{Z}(\cdot, t), t)\right)\right) \circ(\mathbb{I}-Z)+\frac{1}{2}\left[\overline{\widetilde{Z}} f_{z}^{\prime}(\cdot+\widetilde{Z}(\cdot, t), t)\right],
\end{aligned}
$$

transforms the system $(10,11)$ satisfied for $(z, t) \in \Omega_{\widetilde{Z}}$ into the system $(19,21)$. Assuming that $U$ and $G$ are defined for $z$ in $\mathbb{C}_{\delta}$, this system gives, at cubic order, the system

$$
\begin{aligned}
U_{t}^{\prime}+G_{z}^{\prime} & =\frac{1}{2} U_{z}^{\prime}[D]+U_{z}^{\prime} \mathcal{T}\left(D+\left(\bar{U} G_{z}^{\prime}\right)_{x}^{\prime}\right)-G_{z}^{\prime}\left(\mathcal{T}\left(\bar{U} U_{z}^{\prime}\right)\right)_{z}^{\prime}+ \\
& +\left(\mathcal{T}\left\{\bar{U}\left(D+U_{z}^{\prime} G_{z}^{\prime}\right)\right\}\right)_{z}^{\prime}+O(4)
\end{aligned}
$$




$$
\begin{aligned}
G_{t}^{\prime}-i(1+\mu) U & =-G_{z}^{\prime} \mathcal{T}\left(\bar{U} G_{z}^{\prime}\right)_{x}^{\prime}+G_{z}^{\prime}\left(\mathcal{T} D+\frac{1}{2}[D]\right)+ \\
& +\mathcal{T}\left\{\bar{U}\left(G_{z}^{\prime} G_{z z}^{\prime \prime}+i(1+\mu) U_{z}^{\prime} U_{z}^{\prime}\right)\right\}+ \\
& +\frac{1}{2}\left[\bar{U}\left(G_{z}^{\prime} G_{z z}^{\prime \prime}+i(1+\mu) U_{z}^{\prime} U_{z}^{\prime}\right)\right]+ \\
& -\mathcal{T}\left(G_{z}^{\prime} D-(\bar{U} C)_{x}^{\prime}\right)-\frac{1}{2}\left[G_{z}^{\prime} D\right]+O(4),
\end{aligned}
$$

where

$$
\begin{aligned}
& D(x, t)=\bar{G}_{z}^{\prime}(x, t) U_{z}^{\prime}(x, t)-G_{z}^{\prime}(x, t) \bar{U}_{z}^{\prime}(x, t)+O(4), \\
& C(x, t)=\left|G_{z}^{\prime}(x, t)\right|^{2},
\end{aligned}
$$

the space average $[U]$ is constant, and $O(4)$ means terms of order 4 and higher in $(U, G)$ and derivatives of $(U, G)$ near 0.

Remark: More precision with estimates in suitable norms is necessary when trying to solve the existence problem. It is however clear that the right hand side of the system $(17,18)$ has less regularity than the left hand side, since it has derivatives of higher order than are to be found in the linear left hand side. This strongly suggests that an argument like Nash Moser theorem would be at least necessary for solving the existence problem. In the present work, we don't need these estimates, since we are interested in finding solutions as power series of a small amplitude $(\varepsilon=2 \sqrt{\mu})$, with no result on their convergence.

Proof of the lemma: Using (16) in equation (10) leads to

$$
\begin{aligned}
0 & =G_{z}^{\prime}+U_{t}^{\prime}+\left(1-Z_{z}^{\prime}\right) \mathcal{T}\left(\overline{\widetilde{Z}} f_{z}^{\prime}(\xi+\widetilde{Z}(\xi, t), t)\right)_{\xi}^{\prime} \circ(\mathbb{I}-Z)+\frac{1}{2}\left[\left(\overline{\widetilde{Z}} \widetilde{Z}_{\xi}^{\prime}\right)_{t}^{\prime}\right]+ \\
& +\mathcal{T}\left(\widetilde{\widetilde{Z}} \widetilde{Z}_{\xi}^{\prime}\right)_{t}^{\prime} \circ(\mathbb{I}-Z)-Z_{t}^{\prime} \mathcal{T}\left(\widetilde{\widetilde{Z}} \widetilde{Z}_{\xi}^{\prime}\right)_{\xi}^{\prime} \circ(\mathbb{I}-Z)+ \\
& +\left(1-Z_{z}^{\prime}\right)(\mathcal{T} \widetilde{D}) \circ(\mathbb{I}-Z)+\left(1-Z_{z}^{\prime}\right) \frac{1}{2}[\widetilde{D}],
\end{aligned}
$$

A manipulation in the spirit of the previous section and use of property (14) in the form

$$
\begin{aligned}
f_{z}^{\prime}(\xi+\widetilde{Z}(\xi, t), t)+\widetilde{Z}_{t}^{\prime}(\xi, t) & =-(\mathcal{T} \widetilde{D})(\xi, t)-\frac{1}{2}[\widetilde{D}](t)+ \\
& -\widetilde{Z}_{\xi}^{\prime}(\xi, t) f_{z}^{\prime}(\xi+\widetilde{Z}(\xi, t), t)
\end{aligned}
$$

with lemmas $1(\mathrm{~d}), 4(\mathrm{~b})$, definition(8) and the periodicity of $\widetilde{Z}$ yields

$$
\begin{aligned}
0 & =G_{z}^{\prime}+U_{t}^{\prime}-Z_{z}^{\prime} \mathcal{T}\left\{\widetilde{D}+\left(\widetilde{\widetilde{Z}} f_{z}^{\prime}(\xi+\widetilde{Z}(\xi, t), t)\right)_{\xi}^{\prime}\right\} \circ(\mathbb{I}-Z)+ \\
& -\mathcal{T}\left(\widetilde{\widetilde{Z}}\left\{\widetilde{D}+\widetilde{Z}_{\xi}^{\prime} f_{z}^{\prime}(\xi+\widetilde{Z}(\xi, t), t)\right\}\right)_{\xi}^{\prime} \circ(\mathbb{I}-Z)-Z_{z}^{\prime} \frac{1}{2}[\widetilde{D}]+ \\
& -Z_{t}^{\prime} \mathcal{T}\left(\widetilde{\widetilde{Z}} \widetilde{Z}_{\xi}^{\prime}\right)_{\xi}^{\prime} \circ(\mathbb{I}-Z) .
\end{aligned}
$$


This equation is of course implicit in the unknown functions $G$ and $U$, but it has the advantage of being exact, i.e. without approximation. We observe from $(19)$ that the space average $[U]$ is constant in $t$, while $[Z]$ is not constant in $t$. We can now write explicitely this new equation up to cubic terms:

$$
\begin{aligned}
G_{z}^{\prime}+U_{t}^{\prime} & =U_{z}^{\prime} \mathcal{T}\left\{D+\left(\bar{U} G_{z}^{\prime}\right)_{x}^{\prime}\right\}+U_{z}^{\prime} \frac{1}{2}[D]+ \\
& +\mathcal{T}\left(\bar{U}\left\{D+U_{z}^{\prime} G_{z}^{\prime}\right\}\right)_{x}^{\prime}+U_{t}^{\prime} \mathcal{T}\left(\bar{U} U_{z}^{\prime}\right)_{x}^{\prime}+O(4)
\end{aligned}
$$

where

$$
D(x, t)=U_{t}^{\prime}(x, t) \bar{U}_{z}^{\prime}(x, t)-\bar{U}_{t}^{\prime}(x, t) U_{z}^{\prime}(x, t),
$$

which may also be written as (17).

Considering now equation (11), we proceed in the same way to obtain successively

$$
\begin{aligned}
0 & =G_{t}^{\prime}-i(1+\mu) U-Z_{t}^{\prime} \mathcal{T}\left(\widetilde{\widetilde{Z}} f_{z}^{\prime}(\xi+\widetilde{Z}(\xi, t), t)\right)_{\xi}^{\prime} \circ(1-Z)+ \\
& +\mathcal{T}\left(\left(\overline{\widetilde{Z}} f_{z}^{\prime}(\xi+\widetilde{Z}(\xi, t), t)\right)_{t}^{\prime}-i(1+\mu) \overline{\widetilde{Z}} \widetilde{Z}_{\xi}^{\prime}+\widetilde{C}\right) \circ(1-Z)+ \\
& +\frac{1}{2}\left[\left(\overline{\widetilde{Z}} f_{z}^{\prime}(\xi+\widetilde{Z}(\xi, t), t)\right)_{t}^{\prime}-i(1+\mu) \overline{\widetilde{Z}} \widetilde{Z}_{\xi}^{\prime}+\widetilde{C}\right]
\end{aligned}
$$

from which it follows that

$$
\begin{aligned}
0 & =G_{t}^{\prime}-i(1+\mu) U-Z_{t}^{\prime} \mathcal{T}\left(\widetilde{\widetilde{Z}} f_{z}^{\prime}(\xi+\widetilde{Z}(\xi, t), t)\right)_{\xi}^{\prime} \circ(1-Z)+ \\
& +\mathcal{T}(\widetilde{E}) \circ(1-Z)+\frac{1}{2}[\widetilde{E}]
\end{aligned}
$$

where

$$
\begin{gathered}
\widetilde{E} \stackrel{\text { def }}{=}\left(\widetilde{\widetilde{Z}} f_{z}^{\prime}(\xi+\widetilde{Z}(\xi, t), t)\right)_{t}^{\prime}-i(1+\mu) \widetilde{\widetilde{Z}} \widetilde{Z}_{\xi}^{\prime}+\widetilde{C} \\
=\overline{\widetilde{Z}}\left\{f_{z t}^{\prime \prime}(\xi+\widetilde{Z}(\xi, t), t)-i(1+\mu) \widetilde{Z}_{\xi}^{\prime}\right\}+\widetilde{Z} \\
f_{z}^{\prime}\left(\xi+\widetilde{Z} f_{z z}^{\prime \prime}(\xi+\widetilde{Z}(\xi, t), t)+\right. \\
\left.f_{z}^{\prime}(\xi+\widetilde{Z}(\xi, t), t)+\widetilde{Z}_{t}^{\prime}\right\} .
\end{gathered}
$$

We observe that (using lemma $1(\mathrm{~b})$, and the fact that $\widetilde{C}$ is real-valued)

$$
\begin{aligned}
{\left[\overline{\widetilde{Z}}(\mathcal{T} \widetilde{C})_{\xi}^{\prime}+\overline{\widetilde{Z}}_{\xi}^{\prime} \widetilde{C}\right] } & =\left[\overline{\widetilde{Z}}_{\xi}^{\prime}(\widetilde{C}-\mathcal{T} \widetilde{C})\right] \\
& =\left[\overline{\widetilde{Z}}_{\xi}^{\prime}([\widetilde{C}]+\overline{\mathcal{T} \widetilde{C}})\right] \\
& =\overline{\left[\widetilde{Z}_{\xi}^{\prime} \mathcal{T} \widetilde{C}\right]}=0,
\end{aligned}
$$


where the last identity follows by Cauchy's integral formula. Since $D$ is imaginary, lemma $1(\mathrm{~b})$ gives that

$$
\overline{\widetilde{D}}-\overline{\mathcal{T} \widetilde{D}}=[\overline{\widetilde{D}}]-\mathcal{T} \widetilde{D}
$$

which is analytic in $\xi$, and so

$$
\begin{aligned}
{\left[f_{z}^{\prime}(\xi+\widetilde{Z}(\xi, t), t) \overline{\mathcal{T} \widetilde{D}}\right] } & =\left[f_{z}^{\prime}(\xi+\widetilde{Z}(\xi, t), t) \overline{\widetilde{D}}\right] \\
& =-\left[f_{z}^{\prime}(\xi+\widetilde{Z}(\xi, t), t) \widetilde{D}\right]
\end{aligned}
$$

Differentiation of (11) along with (6) gives that

$$
f_{z t}^{\prime \prime}(\xi+\widetilde{Z}(\xi, t), t)-i(1+\mu) \widetilde{Z}_{\xi}^{\prime}=-(\mathcal{T} \widetilde{C})_{\xi}^{\prime}-\widetilde{Z}_{\xi}^{\prime} f_{z t}^{\prime \prime}(\xi+\widetilde{Z}(\xi, t), t) .
$$

From this identity and (10) it follows that

$$
\begin{aligned}
& 0=G_{t}^{\prime}-i(1+\mu) U-Z_{t}^{\prime} \mathcal{T}\left(\overline{\widetilde{Z}} f_{z}^{\prime}(\xi+\widetilde{Z}(\xi, t), t)\right)_{\xi}^{\prime} \circ(1-Z)+ \\
& -\mathcal{T}\left(\overline{\widetilde{Z}}\left\{\widetilde{Z}_{\xi}^{\prime} f_{z t}^{\prime \prime}(\xi+\widetilde{Z}(\xi, t), t)+(\mathcal{T} \widetilde{C})_{\xi}^{\prime}\right\}\right) \circ(1-Z)+ \\
& -\mathcal{T}\left(f_{z}^{\prime}(\xi+\widetilde{Z}(\xi, t), t)\left\{\overline{\mathcal{T} \widetilde{D}+\widetilde{Z}_{\xi}^{\prime} f_{z}^{\prime}(\xi+\widetilde{Z}(\xi, t), t)+\frac{1}{2}[\widetilde{D}]}\right\}\right) \circ(1-Z)+ \\
& +\mathcal{T}\left(\widetilde{Z}_{t}^{\prime} \overline{\widetilde{Z}} f_{z z}^{\prime \prime}(\xi+\widetilde{Z}(\xi, t), t)\right) \circ(1-Z)+ \\
& +\frac{1}{2}\left[\widetilde{Z}\left(\widetilde{Z}_{t}^{\prime} f_{z z}^{\prime \prime}(\xi+\widetilde{Z}(\xi, t), t)-\widetilde{Z}_{\xi}^{\prime} f_{z t}^{\prime \prime}(\xi+\widetilde{Z}(\xi, t), t)\right)\right]+ \\
& +\frac{1}{2}\left[f_{z}^{\prime}(\xi+\widetilde{Z}(\xi, t), t) \widetilde{D}\right] \text {, }
\end{aligned}
$$

and finally

$$
\begin{aligned}
0 & =G_{t}^{\prime}-i(1+\mu) U-Z_{t}^{\prime} \mathcal{T}\left(\widetilde{\widetilde{Z}} f_{z}^{\prime}(\xi+\widetilde{Z}(\xi, t), t)\right)_{\xi}^{\prime} \circ(1-Z)-f_{z}^{\prime} \frac{1}{2}[\widetilde{D}]+ \\
& +\mathcal{T}\left\{\widetilde{\widetilde{Z}}\left(\widetilde{Z}_{t}^{\prime} f_{z z}^{\prime \prime}(\xi+\widetilde{Z}(\xi, t), t)-\widetilde{Z}_{\xi}^{\prime} f_{z t}^{\prime \prime}(\xi+\widetilde{Z}(\xi, t), t)\right)\right\} \circ(1-Z)+ \\
& -f_{z}^{\prime}(\mathcal{T} \widetilde{D}) \circ(1-Z)+\mathcal{T}\left(f_{z}^{\prime}(\xi+\widetilde{Z}(\xi, t), t) \widetilde{D}-(\widetilde{\widetilde{Z}} \widetilde{C})_{\xi}^{\prime}\right) \circ(1-Z)+ \\
& +\frac{1}{2}\left[\widetilde{Z}\left(\widetilde{Z}_{t}^{\prime} f_{z z}^{\prime \prime}(\xi+\widetilde{Z}(\xi, t), t)-\widetilde{Z}_{\xi}^{\prime} f_{z t}^{\prime \prime}(\xi+\widetilde{Z}(\xi, t), t)\right)\right]+ \\
& +\frac{1}{2}\left[f_{z}^{\prime}(\xi+\widetilde{Z}(\xi, t), t) \widetilde{D}\right],
\end{aligned}
$$


This is an exact implicit equation for $G, U$ and $\mu$ only, which has no quadratic term. Up to cubic order it is precisely the following

$$
\begin{aligned}
G_{t}^{\prime}-i(1+\mu) U & =U_{t}^{\prime} \mathcal{T}\left(\bar{U} G_{z}^{\prime}\right)_{x}^{\prime}+G_{z}^{\prime}\left(\mathcal{T} D+\frac{1}{2}[D]\right)+ \\
& -\mathcal{T}\left\{\bar{U}\left(U_{t}^{\prime} G_{z z}^{\prime \prime}-U_{z}^{\prime} G_{z t}^{\prime \prime}\right)\right\}-\frac{1}{2}\left[\bar{U}\left(U_{t}^{\prime} G_{z z}^{\prime \prime}-U_{z}^{\prime} G_{z t}^{\prime \prime}\right)\right]+ \\
& -\mathcal{T}\left(G_{z}^{\prime} D-(\bar{U} C)_{x}^{\prime}\right)-\frac{1}{2}\left[G_{z}^{\prime} D\right]+O(4)
\end{aligned}
$$

This can also be written as (18).

\section{Other representation of the cubic terms}

In this section we derive another explicit form of the system $(17,18)$, using Fourier series, in the spirit of [6]. We are lead naturally to Fourier series because of the structure of the kernel of the linearized operator

$$
\begin{aligned}
U_{t}^{\prime}+G_{z}^{\prime} & =0, \\
G_{t}^{\prime}-i U & =0 .
\end{aligned}
$$

Let us define the Fourier series for $U$ and $G$ :

$$
\begin{aligned}
& U(z, t)=i \sum_{p \geq 0} a_{p}(t) e^{-i p z}, \\
& G(z, t)=\sum_{p \geq 0} b_{p}(t) e^{-i p z} .
\end{aligned}
$$

Because of the symmetry properties

$$
U(-\bar{z}, t)=-\bar{U}(z, t), \quad G(-\bar{z}, t)=\bar{G}(z, t),
$$

the coefficients $a_{p}$ and $b_{p}$ are real functions of $t$. We notice that $a_{0}=[U]$, which is a constant by lemma 5 . Moreover, we assume that

$$
\begin{aligned}
& a_{p} \text { is even in } t, \\
& b_{p} \text { is odd in } t,
\end{aligned}
$$

since we are restricting our attention to solutions of $(1,2)$ with $h(x, t)$ even in $x$ and $t$, as in [8],[1],[6].

Definition 6 We denote by $\mathcal{E}$ the linear space of $2 \pi$-periodic, smooth functions taking values in $\left(\mathbb{R}^{\mathbb{N} \cup\{0\}}\right)^{2}$, where the first element is even, the second being odd, and denote by $\mathcal{F}$ the space of $2 \pi$-periodic, smooth functions taking values in $\left(\mathbb{R}^{\mathbb{N} \cup\{0\}}\right)^{2}$, where the first element is odd, the second being even. 
For any $(A, B) \in \mathcal{E}$, let $A=\left\{a_{p}\right\}_{p \geq 0}, B=\left\{b_{p}\right\}_{p \geq 0}$ and

$$
(\partial B)_{p}=p b_{p} .
$$

Now, the system $(17,18)$ takes the form

$$
\begin{aligned}
\dot{A}-\partial B & =P_{3}(A, A ; B)+R_{A}(A, B), \\
\dot{B}+(1+\mu) A & =Q_{3}(B, B ; A)+(1+\mu) M_{3}(A, A, A)+R_{B}(A, B, \mu),
\end{aligned}
$$

where $P_{3}$ and $Q_{3}$ are bilinear, symmetric with respect to their two first arguments, and linear with respect to the third, while $M_{3}$ is trilinear and symmetric in all its arguments. The remainder terms, $R_{A}$ and $R_{B}$ represent terms of order at least 4 with respect to $(A, B)$. Below we give explicit formulas for the cubic terms $P_{3}, Q_{3}, M_{3}$ (they are different from the ones obtained in [6] since the variables are different). The identification of sequences corresponding to each term in $(17,18)$ is made in Appendix A. We obtain, for $p \geq 0$,

$$
\begin{aligned}
P_{3}(A, A ; B)_{p} & =\sum_{q \geq 1} p q^{2} a_{p} a_{q} b_{q}+\sum_{\substack{p+q=j+r \\
r \geq q+1}} q r j a_{j}\left(a_{q} b_{r}+a_{r} b_{q}\right)+ \\
& -\sum_{\substack{r=p+q+j\\
} q r a_{j}\left(a_{q} b_{r}+a_{r} b_{q}\right)+\sum_{p+q=j+r} p j r a_{q} a_{r} b_{j}+} i a_{\substack{p+q=j+r \\
r \geq q+1}} j r(r-q) a_{q}\left(a_{j} b_{r}-a_{r} b_{j}\right) .
\end{aligned}
$$

Thus $P_{3}(A, A ; B)_{0}=0$. For $p \geq 1$,

$$
\begin{aligned}
Q_{3}(B, B ; A)_{p} & =\sum_{q \geq 1} p q^{2} a_{q} b_{p} b_{q}+\sum_{\substack{p+q=j+r \\
r \geq q+1}} q r j b_{j}\left(a_{q} b_{r}+a_{r} b_{q}\right)+ \\
& +\sum_{\substack{p+q=j+r \\
q \geq r+1}} j r(r-q) a_{q} b_{r} b_{j}-\sum_{p+q=j+r} j q r a_{r} b_{j} b_{q}+ \\
& -\sum_{r=p+q+j} p q r a_{j} b_{q} b_{r}, \\
& M_{3}(A, A, A)_{p}=\sum_{p+q=j+r} j r a_{j} a_{q} a_{r}
\end{aligned}
$$

holds, and for $p=0$,

$$
\begin{aligned}
& Q_{3}(B, B ; A)_{0}=\frac{1}{2} \sum_{j, r \geq 1} j r^{2} a_{j+r} b_{r} b_{j}-j r(r+j) b_{j}\left(a_{r+j} b_{r}+a_{r} b_{r+j}\right), \\
& M_{3}(A, A, A)_{0}=\frac{1}{2} \sum_{r, j \geq 1} j r a_{j} a_{r} a_{r+j}
\end{aligned}
$$


holds. We observe that, for $p=0$

$$
\begin{aligned}
\dot{a}_{0} & =0, \\
\dot{b}_{0}+(1+\mu) a_{0} & =Q_{3}(B, B ; A)_{0}+(1+\mu) M_{3}(A, A, A)_{0}+R_{B}(A, B, \mu)_{0} .
\end{aligned}
$$

Thismeans that $a_{0}$ (const) and $b_{0}$ (odd) can be written as the sums of cubic terms in the other coordinates $(A, B)_{p}, p \geq 1$, plus terms of order 4 in all the variables. Thanks to this uncoupling, at cubic order we can consider the components $p \geq 1$ of system (23) as functions only of $(A, B)_{j}, j \geq 1$. We see below that the essential part of (23) is indeed the cubic part.

\section{$7 \quad$ Study of the linear operator for $\mu=0$}

We define the linear operator $\mathcal{L}$ from $\mathcal{E}$ to $\mathcal{F}$ by

$$
\mathcal{L} X=(\dot{A}-\partial B, \dot{B}+A),
$$

for any $X=(A, B) \in \mathcal{E}$. This linear operator occurs in the principal linear part of (23). The aim of this section is to study the inverse of this operator.

It is clear that

$$
\begin{aligned}
\operatorname{ker}(\mathcal{L}) & =\left\{X=(A, B) \in \mathcal{E} ;(A, B)_{p}=0 \text { if } p \neq q^{2}, q \in \mathbb{N},\right. \\
X_{q^{2}} & \left.=A_{q^{2}}\left(\cos q t,-q^{-1} \sin q t\right), q \in \mathbb{N}, A_{q^{2}} \in \mathbb{R}\right\}
\end{aligned}
$$

$\operatorname{Range}(\mathcal{L})=\left\{Z=(F, G) \in \mathcal{F} ;(F, G)_{q^{2}}=\left(f_{q^{2}}, g_{q^{2}}\right), f_{q^{2}}^{(q)}+q g_{q^{2}}^{(q)}=0, q \in \mathbb{N}\right\}$, where $f_{q^{2}}^{(q)}$ is the $q$ th Fourier coefficient of $f_{q^{2}}$. Notice that $p=0$ does not give any component in $\operatorname{ker}(\mathcal{L})$.

Lemma 7 If $Z \in$ Range $(\mathcal{L})$, there exists a unique $X \in \mathcal{E}$, such that $\mathcal{L} X=$ $Z$ and $X_{q^{2}}=\left(a_{q^{2}}, b_{q^{2}}\right)$ satisfies $-q a_{q^{2}}^{(q)}+b_{q^{2}}^{(q)}=0$ for all $q \in \mathbb{N}$ (we say $\left.X \in \operatorname{ker}(\mathcal{L})^{\perp}\right)$. This defines the pseudo-inverse $\widetilde{\mathcal{L}}^{-1}$ of $\mathcal{L}$ on its range. Every component $X_{p}$ results from a bounded linear operator acting only on $Z_{p}$ (the norm of this operator depends on $p$ ).

Indeed, let us define a projection $P_{0}$ of $\mathcal{F}$ onto the subspace "orthogonal" to Range $(\mathcal{L})$ :

$$
\begin{aligned}
\left(P_{0} Z\right)_{p \neq q^{2}} & =0, \quad\left(P_{0} Z\right)_{q^{2}}=\frac{l_{q^{2}}(Z)}{1+q^{2}}(\sin q t, q \cos q t), q \in \mathbb{N}, \\
l_{q^{2}}(Z) & =\frac{1}{\pi} \int_{0}^{2 \pi}\left[f_{q^{2}}(t) \sin q t+q g_{q^{2}}(t) \cos q t\right] d t .
\end{aligned}
$$


Then $P_{0} Z=0$ if and only if $Z \in \operatorname{Range}(\mathcal{L})$ and $\mathcal{L} X=Z$ where $X=$ $(A, B) \in \operatorname{ker}(\mathcal{L})^{\perp}$ is given for $p \neq q^{2}$ by

$$
\begin{aligned}
a_{p}(t) & =\frac{1}{2 \sin \sqrt{p} \pi} \int_{0}^{2 \pi}\left[\operatorname{sgn}(t-s) f_{p}(s) \sin \sqrt{p}(\pi-|t-s|)\right. \\
& \left.+\sqrt{p} g_{p}(s) \cos \sqrt{p}(\pi-|t-s|)\right] d s, \\
b_{p}(t)= & \frac{1}{2 \sqrt{p} \sin \sqrt{p} \pi} \int_{0}^{2 \pi}\left[\operatorname{sgn}(t-s) \sqrt{p} g_{p}(s) \sin \sqrt{p}(\pi-|t-s|)\right. \\
- & \left.f_{p}(s) \cos \sqrt{p}(\pi-|t-s|)\right] d s,
\end{aligned}
$$

and, for $p=q^{2}$, and $m \neq q$

$$
\begin{aligned}
& a_{q^{2}}^{(m)}=-\left(m^{2}-q^{2}\right)^{-1}\left(m f_{q^{2}}^{(m)}+q^{2} g_{q^{2}}^{(m)}\right), \\
& b_{q^{2}}^{(m)}=\left(m^{2}-q^{2}\right)^{-1}\left(f_{q^{2}}^{(m)}+m g_{q^{2}}^{(m)}\right),
\end{aligned}
$$

while for $m=q$

$$
b_{q^{2}}^{(q)}=\frac{-1}{\left(1+q^{2}\right)} f_{q^{2}}^{(q)}=\frac{q}{1+q^{2}} g_{q^{2}}^{(q)}=q a_{q^{2}}^{(q)},
$$

holds. The result of the lemma follows directly from the above expressions. A difficulty, due to the fact that $\sin \sqrt{p} \pi$ can reach very small values as $p$ increases, results in a (small) loss of regularity in inverting this linear operator $\mathcal{L}$.

\section{The bifurcation problem}

We now show that the elimination of quadratic terms by a suitable choice of variables in section 4 , leads to a bifurcation equation where all critical modes are uncoupled up to cubic order. This clarifies all possible bifurcating (formal) solutions of the standing wave problem. A big difference with the approach in [6] is that here it is not necessary to proceed via the cubic normal form. Such a normal form is presented in Appendix B, giving the solution of an interesting algebraic problem, whose result is however not easily adapted for use here. Another reason for avoiding the cubic normal form is to make it easier to obtain precise estimates on the loss of regularity of the right hand side of (23). These estimates are required, for example, if we want to use the Nash Moser implicit function theorem.

Considering the system (23), we finally obtained a system in the space $\mathcal{F}$ of the form

$$
\mathcal{L} X=\mu \mathcal{J} X+\mathcal{N}\left(X^{(3)} ; \mu\right)+\mathcal{R}(X ; \mu)
$$

where

$$
X=(A, B) \in \mathcal{E}, \quad \mathcal{J} X=(0,-A) \in \mathcal{F},
$$




$$
\mathcal{N}\left(X^{(3)} ; \mu\right)=\left(P_{3}(A, A ; B), Q_{3}(B, B ; A)+(1+\mu) M_{3}(A, A, A)\right),
$$

and

$$
\mathcal{R}(X ; \mu)=\left(R_{A}(A, B), R_{B}(A, B, \mu)\right)=O(4) .
$$

The idea is to use the Lyapunov-Schmidt method.

Let us decompose $X \in \mathcal{E}$ as

$$
X=X_{0}+Y, \quad X_{0} \in \operatorname{Ker}(\mathcal{L}), \quad Y \in \operatorname{Ker}(\mathcal{L})^{\perp} .
$$

Then equation (25) becomes

$$
\begin{aligned}
& \widetilde{\mathcal{L}} Y=\mu\left(\mathbb{I}-P_{0}\right) \mathcal{J}\left(X_{0}+Y\right)+\left(\mathbb{I}-P_{0}\right) \mathcal{N}\left(\left(X_{0}+Y\right)^{(3)} ; \mu\right)+ \\
&+\left(\mathbb{I}-P_{0}\right) \mathcal{R}\left(X_{0}+Y ; \mu\right), \\
& \mu P_{0} \mathcal{J}\left(X_{0}+Y\right)+P_{0} \mathcal{N}\left(\left(X_{0}+Y\right)^{(3)} ; \mu\right)+P_{0} \mathcal{R}\left(X_{0}+Y ; \mu\right)=0 .
\end{aligned}
$$

Since $\widetilde{\mathcal{L}}$ is invertible, and the right hand side is analytic in its arguments, one can formally solve equation (26) with respect to $Y$ as "powers series" of $X_{0}$ and $\mu$ for $X_{0}, \mu$ close to $(0,0)$ in $(\operatorname{ker} \mathcal{L}) \times \mathbb{R}$. In this way we obtain

$$
\begin{aligned}
Y & =\mathcal{Y}\left(X_{0}, \mu\right)=\mu \widetilde{\mathcal{L}}^{-1}\left(\mathbb{I}-P_{0}\right) \mathcal{J} X_{0}+\widetilde{\mathcal{L}}^{-1}\left(\mathbb{I}-P_{0}\right) \mathcal{N}\left(X_{0}^{(3)} ; 0\right)+ \\
& +O\left(\left|\mu^{2}\right|\left|X_{0}\right|+|\mu|\left|X_{0}\right|^{3}+\left|X_{0}\right|^{4}\right)
\end{aligned}
$$

and, after replacing $Y$ by its expression $\mathcal{Y}\left(X_{0}, \mu\right)$ in (27), we have the infinite dimensional bifurcation equation

$$
\mu P_{0} \mathcal{J} X_{0}+P_{0} \mathcal{N}\left(X_{0}^{(3)} ; 0\right)+P_{0} \mathcal{S}\left(X_{0} ; \mu\right)=0,
$$

where

$$
P_{0} \mathcal{S}\left(X_{0} ; \mu\right)=O\left(\left|\mu^{2}\right|\left|X_{0}\right|+|\mu|\left|X_{0}\right|^{3}+\left|X_{0}\right|^{4}\right) .
$$

More precisely, denoting the $j^{2}$ components of $X_{0}$ by $\left(X_{0}\right)_{j^{2}}=\left(a_{j^{2}}, b_{j^{2}}\right)$, we have, for any $q \geq 1$

$$
\begin{aligned}
& P_{3}(A, A ; B)_{q^{2}}=\sum_{j \geq 1} q^{2} j^{4} a_{q^{2}} a_{j^{2}} b_{j^{2}}+\sum_{\substack{q^{2}+m^{2}=j^{2}+r^{2} \\
r^{2} \geq m^{2}+1}} m^{2} r^{2} j^{2} a_{j^{2}}\left(a_{m^{2}} b_{r^{2}}+a_{r^{2}} b_{m^{2}}\right)+ \\
& -\sum_{r^{2}=q^{2}+m^{2}+j^{2}} q^{2} m^{2} r^{2} a_{j^{2}}\left(a_{m^{2}} b_{r^{2}}+a_{r^{2}} b_{m^{2}}\right)+\sum_{q^{2}+m^{2}=j^{2}+r^{2}} q^{2} j^{2} r^{2} a_{m^{2}} a_{r^{2}} b_{j^{2}}+ \\
& +\sum_{\substack{q^{2}+m^{2}=j^{2}+r^{2} \\
r^{2} \geq m^{2}+1}} j^{2} r^{2}\left(r^{2}-m^{2}\right) a_{m^{2}}\left(a_{j^{2}} b_{r^{2}}-a_{r^{2}} b_{j^{2}}\right),
\end{aligned}
$$




$$
\begin{aligned}
Q_{3}(B, B ; A)_{q^{2}}= & \sum_{j \geq 1} q^{2} j^{4} a_{j^{2}} b_{q^{2}} b_{j^{2}}+\sum_{\substack{q^{2}+m^{2}=j^{2}+r^{2} \\
r^{2} \geq m^{2}+1}} q r j b_{j}\left(a_{q} b_{r}+a_{r} b_{q}\right)+ \\
& -\sum_{q^{2}+m^{2}=j^{2}+r^{2}} j^{2} m^{2} r^{2} a_{r^{2}} b_{j^{2}} b_{m^{2}}-\sum_{r^{2}=q^{2}+m^{2}+j^{2}} q^{2} m^{2} r^{2} a_{j^{2}} b_{m^{2}} b_{r^{2}}+ \\
& +\sum_{\substack{q^{2}+m^{2}=j^{2}+r^{2} \\
m^{2} \geq r^{2}+1}} j^{2} r^{2}\left(r^{2}-m^{2}\right) a_{m^{2}} b_{r^{2}} b_{j^{2}}, \\
& M_{3}(A, A, A)_{q^{2}}=\sum_{q^{2}+m^{2}=j^{2}+r^{2}} j^{2} r^{2} a_{j^{2}} a_{m^{2}} a_{r^{2}} .
\end{aligned}
$$

Now, for any $q \geq 1$,

$$
\left(a_{q^{2}}, b_{q^{2}}\right)=A_{q^{2}}\left(\cos q t,-q^{-1} \sin q t\right), A_{q^{2}} \in \mathbb{R}
$$

and the computation of $P_{0} \mathcal{N}\left(X_{0}^{(3)} ; 0\right)$ (in particular, for the expression $l_{q^{2}}\left(\mathcal{N}\left(X_{0}^{(3)} ; 0\right)\right.$ ) from (24)), introduces integrals of periodic functions typically as $e^{i( \pm r \pm j \pm m \pm q) t}$, hence the following lemma is useful (proof left to the reader).

Lemma 8 All solutions of

$$
\begin{aligned}
q^{2}+m^{2} & =j^{2}+r^{2}, \\
r \pm j \pm m \pm q & =0, \quad q, m, j, r \geq 1,
\end{aligned}
$$

are given by i) $q=r, j=m$, or ii) $q=j, r=m$.

All solutions of

$$
\begin{gathered}
r^{2}=q^{2}+m^{2}+j^{2} \\
r \pm j \pm m \pm q=0, \quad q, m, j, r \geq 1,
\end{gathered}
$$

are given by i) $q+m=j+r$, or ii) $q+j=r+m$, or iii) $q+r=j+m$.

We use this lemma in the expressions for $P_{3}, Q_{3}, M_{3}$ and write below the terms which may produce non zero terms in the iterm $l_{q^{2}}\left(\mathcal{N}\left(X_{0}^{(3)} ; 0\right)\right)$. We denote these restricted expressions by $P_{3}^{\prime}, Q_{3}^{\prime}, M_{3}^{\prime}$ :

$$
\begin{aligned}
P_{3}^{\prime}(A, A ; B)_{q^{2}} & =2 q^{6} a_{q^{2}}^{2} b_{q^{2}}+\sum_{j \geq q+1} q^{2} j^{2}\left(q^{2}+j^{2}\right) a_{j^{2}} a_{q^{2}} b_{j^{2}}+q^{4} j^{2} a_{j^{2}}^{2} b_{q^{2}}+ \\
& +\sum_{j \leq q-1} 2 q^{4} j^{2} a_{j^{2}}^{2} b_{q^{2}}+3 q^{2} j^{4} a_{j^{2}} a_{q^{2}} b_{j^{2}}+ \\
& -\sum_{(q, j, r, m) \in \mathcal{K}} q^{2} m^{2} r^{2} a_{j^{2}}\left(a_{m^{2}} b_{r^{2}}+a_{r^{2}} b_{m^{2}}\right)
\end{aligned}
$$




$$
\begin{aligned}
& Q_{3}^{\prime}(B, B ; A)_{q^{2}}=-\sum_{j \geq q+1} q^{2} j^{4} a_{q^{2}} b_{j^{2}}^{2}+j^{2} q^{2}\left(q^{2}-j^{2}\right) a_{j^{2}} b_{j^{2}} b_{q^{2}}+ \\
&+\sum_{j \leq q-1} q^{2} j^{4} a_{j^{2}} b_{j^{2}} b_{q^{2}}-\sum_{(q, j, r, m) \in \mathcal{K}} q^{2} m^{2} r^{2} a_{j^{2}} b_{m^{2}} b_{r^{2}} \\
& M_{3}^{\prime}(A, A, A)_{q^{2}}=q^{4} a_{q^{2}}^{3}+\sum_{j \neq q} 2 j^{2} q^{2} a_{j^{2}}^{2} a_{q^{2}}
\end{aligned}
$$

where $(q, j, r, m) \in \mathcal{K}$ means $r^{2}=q^{2}+m^{2}+j^{2}$, and $r \pm j \pm m \pm q=0$. Notice that these expressions are strongly related with the normal form analysis in Appendix B. Now replacing $\left(a_{q^{2}}, b_{q^{2}}\right)$ by its expression, we can compute

$$
l_{q^{2}}\left(\mu \mathcal{J} X_{0}\right)=-\mu q A_{q^{2}},
$$

and

$$
\begin{aligned}
l_{q^{2}}\left(\mathcal{N}\left(X_{0}^{(3)} ; 0\right)\right) & =\frac{1}{\pi} \int_{0}^{2 \pi}\left\{P_{3}^{\prime}(A, A ; B)_{q^{2}} \sin q t+\right. \\
& \left.+q\left(Q_{3}^{\prime}(B, B ; A)_{q^{2}}+M_{3}^{\prime}(A, A, A)_{q^{2}}\right) \cos q t\right\} d t
\end{aligned}
$$

A lengthy, but elementary computation (see Appendix C) shows that

$$
l_{q^{2}}\left(\mathcal{N}\left(X_{0}^{(3)} ; 0\right)\right)=\frac{q^{5} A_{q^{2}}^{3}}{4} .
$$

The result is that (28) may be written as

$$
-\mu q A_{q^{2}}+\frac{q^{5} A_{q^{2}}^{3}}{4}+R_{q}\left(X_{0}, \mu\right)=0, \quad q=1,2, \ldots
$$

with $R_{q}\left(X_{0}, \mu\right)=O\left(\left|\mu^{2}\right|\left|X_{0}\right|+|\mu|\left|X_{0}\right|^{3}+\left|X_{0}\right|^{4}\right)$.

Remark: we may notice here that if we tried to use the same method directly on system $(10,11)$, we should have a quadratic $\mathcal{N}^{\prime}$ but such that $l_{q^{2}}\left(\mathcal{N}^{\prime}\left[X_{0}^{(2)}\right]\right)=0$. This cancellation is because a non-zero contribution would come from an exponential of the type $e^{i( \pm r \pm j \pm q) t}$, with $r^{2}=q^{2}+j^{2}$, and this always gives a zero average $(r, j, q \geq 1)$. Hence it would be needed to compute the term of order $\left\|X_{0}\right\|^{2}$ in $Y$, which has to be inserted into the cubic order terms of the bifurcation equation......and then it would be a terrible mess. So, the suppression of quadratic terms made in section 4 , allows to greatly simplify the computation of the bifurcation equation made here.

Now we make the following rescaling

$$
\mu=\frac{\varepsilon^{2}}{4}, \quad A_{q^{2}}=\frac{\varepsilon \alpha_{q}}{q^{2}}, \quad q=1,2, \ldots
$$


and we define $\xi_{0} \in \mathbb{R}^{\mathbb{N}}$ such that $\left(\xi_{0}\right)_{q^{2}}=\alpha_{q}$. Equation (29) becomes

$$
\alpha_{q}-\alpha_{q}^{3}+\varepsilon S_{q}\left(\xi_{0}, \varepsilon\right)=0, \quad q=1,2, \ldots
$$

where $S_{q}\left(\xi_{0}, \varepsilon\right)$ has a formal powers series in $\varepsilon$, with smooth coefficients as functions of $\xi_{0}$.

Formal solutions of (31) in terms or series in powers of $\varepsilon$, can now be obtained. We observe, of course that $\xi_{0}=0$ is a solution, which corresponds to the flat free surface in the original problem. Let $I \subset \mathbb{N}$ be arbitrary and consider any $\xi_{0}^{0} \in \mathbb{R}^{\mathbb{N}}$ such that $\alpha_{q}=( \pm 1)_{q}$ for $q \in I, \alpha_{q}=0$ for $q \notin I$. Notice that we may choose 1 or -1 arbitrarily, when $q$ varies in $I$, and that the set $I$ may be infinite, $I=\mathbb{N}$ is one of the possibilities. Equation (31) is satisfied when $\varepsilon=0$, for $\xi_{0}=\xi_{0}^{0}$, and since 0 and \pm 1 are simple roots of $\alpha_{q}-\alpha_{q}^{3}=0$, it is clear that one can compute in a standard way the power series

$$
\xi_{0}=\xi_{0}^{0}+\varepsilon \xi_{0}^{1}+\ldots \varepsilon^{k} \xi_{0}^{k}+\ldots
$$

which is a formal solution of (31). Coming back to (25), we then see that $(A, B)=X_{0}+O\left(\varepsilon^{2}\right)$.

We sum up our result by the following

Theorem 9 The system (23) in the space $\mathcal{F}$ admits infinitely many formal solutions $(A, B) \in \mathcal{E}$ in series of powers of an amplitude $\varepsilon$, of the form

$$
\begin{aligned}
(A, B) & =\sum_{n \geq 1} \varepsilon^{n}(A, B)^{(n)}, \text { where }(A, B)^{(n)} \in \mathcal{E}, \\
(A, B)_{p \neq q^{2}}^{(1)} & =0,(A, B)_{q^{2}, q \notin I}^{(1)}=0 \\
(A, B)_{q^{2}, q \in I}^{(1)} & =\left(\frac{( \pm 1)_{q}}{q^{2}} \cos q t, \frac{-( \pm 1)_{q}}{q^{3}} \sin q t\right),
\end{aligned}
$$

where $\varepsilon^{2}=4 \mu$, and $I \subset \mathbb{N}$ is chosen arbitrarily.

Remark 1: Here, as in [1], we have no result on the convergence of these expansions in powers of $\varepsilon$. This is still an open problem, not only for one dominant mode solution, but for all others found here.

Remark 2: The solution obtained by Amick and Toland (called the Stokes solution) corresponds to $I=\{1\}, \alpha_{1}=+1$.

Remark 3: We have in principle a choice between the signs \pm in front of any dominant mode. However many of the choices give the same solutions, after a suitable translation of the time origin.

Remark 4: Coming back to the physical quantities, we observe that the principal parts (order $\sqrt{\mu}$ ) of the complex potential and of the conformal 
$\operatorname{map}(\zeta=z-Z(z, t))$ are now

$$
\begin{aligned}
& f(z, t)=-2 \sqrt{\mu} \sum_{q \in I} \frac{( \pm 1)_{q}}{q^{3}} e^{-i q^{2} z} \sin q t+O(\mu), \\
& Z(z, t)=2 i \sqrt{\mu} \sum_{q \in I} \frac{( \pm 1)_{q}}{q^{2}} e^{-i q^{2} z} \cos q t+O(\mu),
\end{aligned}
$$

which corresponds to the following principal part of the free surface (in the original coordinates)

$$
h(x, t)=2 \sqrt{\mu} \sum_{q \in I} \frac{( \pm 1)_{q}}{q^{2}} \cos q^{2} x \cos q t+O(\mu) .
$$

We notice that the relative values of the dominant modes are in agreement with those found by Bryant and Stiassnie in [2] in the cases $I=\{1,2\}$ and $I=\{1,2,3\}$.

\section{Appendix A}

Calculation of $P_{3}, Q_{3}, M_{3}$ in (23):

$$
\begin{gathered}
\left(\frac{1}{2} U_{z}^{\prime}[D]\right)_{p}=i \sum_{q \geq 1} p q^{2} a_{p} a_{q} b_{q}, \\
\left(U_{z}^{\prime} \mathcal{T} D\right)_{p}=i \sum_{\substack{p+q=j+r \\
r \geq q+1}} q r j a_{j}\left(a_{q} b_{r}+a_{r} b_{q}\right), \\
\left((\mathcal{T} \bar{U} D)_{z}^{\prime}\right)_{p}=-i \sum_{r=p+q+j} p q r a_{j}\left(a_{q} b_{r}+a_{r} b_{q}\right), \\
\left(U_{z}^{\prime} \mathcal{T}\left(\bar{U} G_{z}^{\prime}\right)_{x}^{\prime}\right)_{p}=i \sum_{p+q=j+r} j r(r-q) a_{j} a_{q} b_{r}, \\
-\left(G_{z}^{\prime} \mathcal{T}\left(\bar{U} U_{z}^{\prime}\right)_{x}^{\prime}\right)_{p}=-i \sum_{p+q=j+r} j r(r-q) a_{q} a_{r} b_{j}, \\
\left(\mathcal{T}\left(\bar{U} U_{z}^{\prime} G_{z}^{\prime}\right)_{x}^{\prime}\right)_{p}=i \sum_{p+q=j+r} p j r a_{q} a_{r} b_{j},
\end{gathered}
$$




$$
\begin{aligned}
& \left(\frac{1}{2} G_{z}^{\prime}[D]\right)_{p}=\sum_{q \geq 1} p q^{2} a_{q} b_{p} b_{q}, \\
& \left(G_{z}^{\prime} \mathcal{T} D\right)_{p}=\sum_{p+q=j+r} q r j b_{j}\left(a_{q} b_{r}+a_{r} b_{q}\right), \\
& r \geq q+1 \\
& -\left(G_{z}^{\prime} \mathcal{T}\left(\bar{U} G_{z}^{\prime}\right)_{x}^{\prime}\right)_{p}=-\sum_{\substack{p+q=j+r \\
r \geq q+1}} j r(r-q) a_{q} b_{r} b_{j}, \\
& \left(\mathcal{T}\left(\bar{U} G_{z}^{\prime} G_{z z}^{\prime \prime}\right)\right)_{p}=\sum_{p+q=j+r} j r^{2} a_{q} b_{r} b_{j}, p \geq 1, \\
& \frac{1}{2}\left[\bar{U} G_{z}^{\prime} G_{z z}^{\prime \prime}\right]=\frac{1}{2} \sum_{j, r \geq 1} j r^{2} a_{j+r} b_{r} b_{j}, \\
& -\left(\mathcal{T}\left(G_{z}^{\prime} D\right)\right)_{p}=-\sum_{p+q=j+r} j q r b_{j}\left(a_{q} b_{r}+a_{r} b_{q}\right), p \geq 1, \\
& -\frac{1}{2}\left[G_{z}^{\prime} D\right]=-\frac{1}{2} \sum_{r, j \geq 1} j r(r+j) b_{j}\left(a_{r+j} b_{r}+a_{r} b_{r+j}\right), \\
& \left(\mathcal{T}(\bar{U} C)_{x}^{\prime}\right)_{p}=-\sum_{r=p+q+j} \operatorname{pqra}_{j} b_{q} b_{r} \\
& \left(\mathcal{T}\left(\bar{U} U_{z}^{\prime} U_{z}^{\prime}\right)\right)_{p}=-i \sum_{p+q=j+r} j r a_{j} a_{q} a_{r}, p \geq 1, \\
& \frac{1}{2}\left[\bar{U} U_{z}^{\prime} U_{z}^{\prime}\right]=-\frac{i}{2} \sum_{r, j \geq 1} j r a_{j} a_{r} a_{r+j},
\end{aligned}
$$

are all necessary terms for writing polynomials $P_{3}, Q_{3}, M_{3}$.

\section{Appendix B}

The idea in [6] was to remove non resonant cubic terms in system (23), restricted to components $p \geq 1$, since the components for $p=0$ are uncoupled up to order 4 . We explore the following class of polynomial near-identity changes of variables

$$
\begin{aligned}
& A^{\prime}=A+(1+\mu)^{-1} \gamma(B, B ; A)+\beta(A, A, A), \\
& B^{\prime}=B+\alpha(A, A ; B)+(1+\mu)^{-1} \partial^{-1} \gamma(B, B ; \partial B),
\end{aligned}
$$

where $\alpha, \beta, \gamma$ are trilinear in their arguments, and we may assume that $\alpha$ and $\gamma$ are symmetric with respect to their two first arguments, and $\beta$ is 
symmetric in all three. After such a transformation, the new cubic terms in (23) have the same form and

$$
\begin{aligned}
P_{3}^{\prime}(A, A ; B) & =P_{3}(A, A ; B)-\partial \alpha(A, A ; B)+3 \beta(A, A, \partial B)-2 \gamma(A, B ; A), \\
Q_{3}^{\prime}(B, B ; A) & =Q_{3}(B, B ; A)+2 \alpha(A, \partial B ; B)+\gamma(B, B ; A)+ \\
& -2 \partial^{-1} \gamma(A, B ; \partial B)-\partial^{-1} \gamma(B, B ; \partial A), \\
M_{3}^{\prime}(A, A, A) & =M_{3}(A, A, A)+\beta(A, A, A)-\alpha(A, A ; A),
\end{aligned}
$$

where we notice that the operator $\partial$ is invertible since $p \geq 1$. The idea now is to choose suitable $\alpha, \beta, \gamma$ to maximise the number of terms in $P_{3}^{\prime}, Q_{3}^{\prime}, M_{3}^{\prime}$ that cancel, and only "resonant terms" of $P_{3}, Q_{3}, M_{3}$ remain in the new system at cubic order. So, with $P_{3}^{\prime}, Q_{3}^{\prime}, M_{3}^{\prime}$ regarded as arbitrary given polynomials with the correct symmetry, we try to solve the above system with respect to $\alpha, \beta, \gamma$ and look to the compatibility conditions under which it can be solved. Let us introduce the coefficients of $\alpha \beta$, and $\gamma$ : for any $A, B, C \in \mathbb{R}^{\mathbb{N}}$

$$
\begin{aligned}
& \alpha(A, B ; C)_{p}=\sum_{i, j, k \geq 1} \alpha_{i j k}^{(p)} a_{i} b_{j} c_{k}, \quad \alpha_{i j k}^{(p)}=\alpha_{j i k}^{(p)}, \\
& \gamma(A, B ; C)_{p}=\sum_{i, j, k \geq 1} \gamma_{i j k}^{(p)} a_{i} b_{j} c_{k}, \quad \gamma_{i j k}^{(p)}=\gamma_{j i k}^{(p)}, \\
& \beta(A, B, C)_{p}=\sum_{i, j, k \geq 1} \beta_{i j k}^{(p)} a_{i} b_{j} c_{k}, \quad \beta_{i j k}^{(p)}=\beta_{j i k}^{(p)}=\beta_{i k j}^{(p)}=\beta_{k j i}^{(p)} .
\end{aligned}
$$

We will see that the system decouples into systems of equations for the $i j k$, $j k i, k i j$ components of $\alpha, \beta, \gamma$. In most cases, these systems can be solved without further consideration. However sometimes, in what we call resonant cases, the resulting system is not solvable since a certain determinant is zero. These compatibility conditions provide the "resonant terms", as it is usual in a normal form technique. Then the result of this section is sum up below.

Lemma 10 For $p=q^{2}$, the resonant coefficients are given

(i) by

$$
i=q^{2}, j=k, \quad \text { or } j=q^{2}, i=k \text { or } k=q^{2}, i=j,
$$

(ii) and by (with all permutations)

$$
\begin{aligned}
i & =K^{2}, \\
j & =\frac{1}{4}(Q-q+K)^{2}, \\
k & =\frac{1}{4}(Q+q-K)^{2},
\end{aligned}
$$

where $K \in \mathbb{Z} \backslash\{0\}, Q \in \mathbb{N} \cup\{0\}$ are such that $K+Q$ has the same parity as $q$, and such that $i j k>0$. 
First step of the proof: The symmetric polynomial $\beta$ is completely determined by

$$
\beta(A, A, A)=\alpha(A, A ; A)+M_{3}^{\prime}(A, A, A)-M_{3}(A, A, A),
$$

and the unknown cubic polynomials $\alpha$ and $\gamma$ are now solutions of the system $P_{3}^{\prime \prime}(A, A ; B)=-\partial \alpha(A, A ; B)+\alpha(A, A ; \partial B)+2 \alpha(A, \partial B ; A)-2 \gamma(A, B ; A)$,

$Q_{3}^{\prime \prime}(B, B ; A)=2 \partial \alpha(A, \partial B ; B)+\partial \gamma(B, B ; A)-2 \gamma(A, B ; \partial B)-\gamma(B, B ; \partial A)$,

where

$$
\begin{aligned}
& P_{3}^{\prime \prime}(A, A ; B)=P_{3}^{\prime}(A, A ; B)-P_{3}(A, A ; B)-3\left(M_{3}^{\prime}-M_{3}\right)(A, A, \partial B), \\
& Q_{3}^{\prime \prime}(B, B ; A)=\partial Q_{3}^{\prime}(B, B ; A)-\partial Q_{3}(B, B ; A) .
\end{aligned}
$$

The system (32) is indeed the same as in [6], where there is a mistake in the computation of the compatibility conditions. So we present below a (hopefully correct) way to solve it for $\alpha$ and $\gamma$.

First observe that $(32)_{1}$ is solvable with respect to $\gamma$. Indeed, it is an equation of the form

$$
\gamma(A, B ; A)=F(A, A ; B)
$$

where $F$ is symmetric with respect to its two first arguments, and we look for $\gamma$ symmetric in its two first arguments. The solution of this algebraic equation is given by

$$
\gamma(B, B ; A)=2 F(A, B ; B)-F(B, B ; A)
$$

i.e.

$$
\gamma(B, C ; A)=F(A, B ; C)+F(A, C ; B)-F(B, C ; A)
$$

which allows us to express $\gamma$ in terms of $\alpha$ and of the known polynomials $P_{3}^{\prime \prime}, Q_{3}^{\prime \prime}$. The remaining equation for $\alpha$ is complicated, and it is in fact easier to proceed with components as follows. $(p))$

Equations (32) lead to (for simplicity we now suppress the superscript

$$
\begin{aligned}
p\left(j \alpha_{j k i}+i \alpha_{k i j}\right)-i \gamma_{j k i}-j \gamma_{k i j}+(p-k) \gamma_{i j k} & =q_{i j k}^{\prime \prime}, \\
-p \alpha_{i j k}+k\left(\alpha_{i j k}+\alpha_{j k i}+\alpha_{k i j}\right)-\left(\gamma_{j k i}+\gamma_{k i j}\right) & =p_{i j k}^{\prime \prime},
\end{aligned}
$$

where $p_{i j k}^{\prime \prime}, q_{i j k}^{\prime \prime}$ are the known coefficients of $\left(P_{3}^{\prime \prime}\right)_{p}$ and $\left(Q_{3}^{\prime \prime}\right)_{p}$, and we check that $p_{i j k}^{\prime \prime}=p_{j i k}^{\prime \prime}, q_{i j k}^{\prime \prime}=q_{j i k}^{\prime \prime}$. The determinant of this system, with respect to $\alpha_{i j k}, \alpha_{j k i}, \alpha_{k i j}, \gamma_{i j k}, \gamma_{j k i}, \gamma_{k i j}$ is

$$
\widetilde{\Delta}=\left|\begin{array}{llllll}
0 & p j & p i & p-k & -i & -j \\
p j & 0 & p k & -k & p-i & -j \\
p i & p k & 0 & -k & -i & p-j \\
k-p & k & k & 0 & -1 & -1 \\
i & i-p & i & -1 & 0 & -1 \\
j & j & j-p & -1 & -1 & 0
\end{array}\right| .
$$


We obtain $\gamma$ in terms of $\alpha$ :

$$
2 \gamma_{i j k}=(i+j-k-p)\left(\alpha_{i j k}+\alpha_{j k i}+\alpha_{k i j}\right)+2 p \alpha_{i j k}+p_{i j k}^{\prime \prime}-p_{k i j}^{\prime \prime}-p_{j k i}^{\prime \prime},
$$

and eliminate $\gamma$ to find that

$$
\begin{aligned}
Q_{3}^{\prime \prime \prime}(B, B ; A) & =3 \alpha(A, \partial B ; B)-3 \alpha(B, \partial A ; B)+\alpha(A, B ; \partial B)+ \\
& +\partial \alpha(B, B ; A)-\partial \alpha(A, B ; B)-\alpha(B, B ; \partial A)
\end{aligned}
$$

where

$$
\begin{aligned}
Q_{3}^{\prime \prime \prime}(B, B ; A) & =P_{3}^{\prime \prime}(A, B ; B)-P_{3}^{\prime \prime}(B, B ; A)+ \\
& +\partial^{-1}\left[Q_{3}^{\prime \prime}(B, B ; A)-Q_{3}^{\prime \prime}(A, B ; B)\right] .
\end{aligned}
$$

This leads to

$$
[p-i+3(k-j)] \alpha_{j k i}+[p-j+3(k-i)] \alpha_{k i j}+2(k-p) \alpha_{i j k}=-2 q_{i j k}^{\prime \prime \prime},
$$

where

$$
2 q_{i j k}^{\prime \prime \prime}=p_{k i j}^{\prime \prime}+p_{j k i}^{\prime \prime}-2 p_{i j k}^{\prime \prime}+\frac{1}{p}\left(2 q_{i j k}^{\prime \prime}-q_{j k i}^{\prime \prime}-q_{k i j}^{\prime \prime}\right) .
$$

We also observe that

$$
q_{i j k}^{\prime \prime \prime}+q_{j k i}^{\prime \prime \prime}+q_{k i j}^{\prime \prime \prime}=0
$$

by construction, which means that the system (36) for $\alpha_{i j k}, \alpha_{j k i}, \alpha_{k i j}$ is not sufficient, because we made 3 linearly dependent combinations of the 6 equations (this was the mistake in [6]). To solve the system with respect to $\alpha_{i j k}, \alpha_{j k i}, \alpha_{k i j}$ we take two of the equations in (36) and a third obtained from replacing $\gamma$ by its expression in terms of $\alpha$ in (34). We then arrive at a system whose determinant may be written

$$
\Delta=\left|\begin{array}{lll}
2(k-p) & p-i+3(k-j) & p-j+3(k-i) \\
p-k+3(i-j) & 2(i-p) & p-j+3(i-k) \\
E+4 p(p-k) & E+2 p(p-i+j-k) & E+2 p(p-j+i-k)
\end{array}\right|
$$

where

$$
\begin{aligned}
E & =-3 p^{2}+2 p(i+j+k)+i^{2}+j^{2}+k^{2}-2 i j-2 i k-2 j k \\
& =2\left(i^{2}+j^{2}+k^{2}-p^{2}\right)-(i+j+k-p)^{2} .
\end{aligned}
$$

Let us define

$$
\begin{aligned}
& S_{1}=i+j+k, \\
& S_{2}=i^{2}+j^{2}+k^{2}, \\
& S_{3}=i^{3}+j^{3}+k^{3} .
\end{aligned}
$$


Then we have

$$
E=2\left(S_{2}-p^{2}\right)-\left(S_{1}-p\right)^{2} .
$$

After some computations we find that

$$
\begin{aligned}
\Delta & =3 E\left\{4(p-j)(p-k)-(i-j-k+p)^{2}\right\}+ \\
& -24 p(p+k-i-j)\left[(i-j)^{2}-(p-k)^{2}\right],
\end{aligned}
$$

which appears to be a polynomial in $(i, j, k, p)$ homogeneous of degree 4 , and symmetric in $(i, j, k)$. This becomes

$$
\Delta=-3 E\left[E+8 p\left(S_{1}+p\right)\right]+16 p F,
$$

where

$$
F=4\left(S_{3}-p^{3}\right)-\left(S_{1}-p\right)^{3} .
$$

We observe that

$$
E=F=0
$$

for

$$
i=p \text {, and } j=k \text { or } j=p \text {, and } i=k \text {, or } k=p \text {, and } i=j,
$$

and in these cases the determinant $\Delta$ vanishes (the matrix has rank one). We need now to study all solutions of $\Delta=0$. A first easy result is the following

Lemma 11 For $p=q^{2}$, the solutions $(i, j, k)$ of $\Delta=0$ are given by

$$
\begin{aligned}
& S_{1}=q^{2}+2 I, \\
& S_{2}=q^{4}+2 I^{2}+4 q J, \\
& S_{3}=q^{6}+2 I^{3}+3 J\left(J+q S_{1}+q^{3}\right),
\end{aligned}
$$

where $I$ and $J$ are integers such that the system

$$
\begin{aligned}
& S_{1}=i+j+k, \\
& S_{2}=i^{2}+j^{2}+k^{2}, \\
& S_{3}=i^{3}+j^{3}+k^{3},
\end{aligned}
$$

defines positive integers $i, j, k$.

Proof: From $\Delta=0$, we deduce that if $p=q^{2}$, then $E=4 q A_{1}, F=3 B_{1}$. Hence we have

$$
\begin{aligned}
& 2\left(S_{2}-p^{2}\right)-\left(S_{1}-p\right)^{2}=4 q A_{1}, \\
& 4\left(S_{3}-p^{3}\right)-\left(S_{1}-p\right)^{3}=3 B_{1},
\end{aligned}
$$


which implies the existence of integers $I, J$ and $B_{2}$ such that $A_{1}=2 J$, $B_{1}=4 B_{2}$ and

$$
\begin{aligned}
& S_{1}=q^{2}+2 I, \\
& S_{2}=q^{4}+2 I^{2}+4 q J, \\
& S_{3}=q^{6}+2 I^{3}+3 B_{2}, \\
& B_{2}=J\left[J+q\left(S_{1}+q^{2}\right)\right],
\end{aligned}
$$

which proves the lemma 11.

Proof of lemma 10 concluded: We can then check that if $p=q^{2}$, and $\Delta=0$

$$
\begin{aligned}
i j+j k+k i & =I^{2}+2 q(q I-J), \\
i j k & =(q I-J)^{2},
\end{aligned}
$$

hence $i, j, k$ are the 3 roots of the cubic equation

$$
X^{3}-\left(q^{2}+2 I\right) X^{2}+\left[I^{2}+2 q(q I-J)\right] X-(q I-J)^{2}=0,
$$

which may be written as

$$
\left.X(X-I)^{2}-[q(X-I)+J)\right]^{2}=0 .
$$

(i) If in (38), $X=I$, then $J=0$ and the result (i) of Lemma 10 holds.

(ii) Assume $X \neq I$. Then there exists $K \in \mathbb{Z} \backslash\{0\}$ such that $X=K^{2}$, hence $J=(K-q)\left(K^{2}-I\right)$, and (38) reads

$$
\left(X-K^{2}\right)\left[(X-I)^{2}+\left(K^{2}-q^{2}\right)(X-I)+\left(K^{2}-I\right)(K-q)^{2}\right]=0 .
$$

The two remaining roots need to be integer, hence there is $Q \in \mathbb{N} \cup\{0\}$ such that

$$
(K+q)^{2}-4\left(K^{2}-I\right)=Q^{2} .
$$

In other words

$$
I=K^{2}+\frac{1}{4}\left[Q^{2}-(K+q)^{2}\right],
$$

and the two remaining roots are given by the formulas in lemma 10. This completes the proof.

Lemma 10 above gives all resonant terms for the components $p=q^{2}$ of cubic coefficients $P_{3}, Q_{3}, M_{3}$ which corresponds to the coefficients $p_{i j k}^{\left(q^{2}\right)}$, $q_{i j k}^{\left(q^{2}\right)}, m_{i j k}^{\left(q^{2}\right)}$ we cannot cancel via the previous change of variables. This corrects the wrong result given in section 3 of [6]. However we don't use this normal form here since there are lot of terms not easy to write explicitly. Moreover, the computation of the bifurcation equation may be done without worrying about the non resonant terms. 


\section{Appendix C}

The term $\frac{q^{5}}{4} A_{q^{2}}^{3}$ comes from

$$
\frac{A_{q^{2}}^{3}}{\pi} \int_{0}^{2 \pi}\left(\frac{-2 q^{6}}{q} \cos ^{2}(q t) \sin ^{2}(q t)+q^{5} \cos ^{4}(q t)\right) d t .
$$

Now, for $j \leq q-1$, we have

$$
0=\int_{0}^{2 \pi}\left(\frac{-2 q^{4} j^{2}}{q} \cos ^{2}(j t) \sin ^{2}(q t)+2 j^{2} q^{3} \cos ^{2}(j t) \cos ^{2}(q t)\right) d t,
$$

and for $j \geq q+1$, we have

$$
\begin{aligned}
0 & =\int_{0}^{2 \pi}\left(\frac{-q^{4} j^{2}}{q} \cos ^{2}(j t) \sin ^{2}(q t)-\frac{q^{3} j^{4}}{j^{2}} \sin ^{2}(j t) \cos ^{2}(q t)+\right. \\
& \left.+2 j^{2} q^{3} \cos ^{2}(j t) \cos ^{2}(q t)\right) d t
\end{aligned}
$$

The only non trivial result comes from the computation of $b_{j m r}^{(q)}$ coming from the terms such that $(q, j, r, m) \in \mathcal{K}$ in the expression

$$
\begin{aligned}
& l_{q^{2}}\left(\mathcal{N}\left(X_{0}^{(3)} ; 0\right)\right)=\frac{q^{5}}{4} A_{q^{2}}^{3}+\sum_{(q, j, r, m) \in \mathcal{K}} b_{j m r}^{(q)} A_{j^{2}} A_{m^{2}} A_{r^{2}}, \\
& b_{j m r}^{(q)}=\frac{q^{2} m r}{\pi} \int_{0}^{2 \pi} \cos j t(-q \cos q t \sin m t \sin r t+ \\
& \quad+m \sin q t \cos m t \sin r t+r \sin q t \sin m t \cos r t) d t .
\end{aligned}
$$

In fact we can consider the symmetrized version $b_{j m r}^{(q)}+b_{m j r}^{(q)}$ since the expression is symmetric in $(j, m)$. Hence we have

$$
\begin{aligned}
b_{j m r}^{(q)}+b_{m j r}^{(q)} & =\frac{q^{2} r}{\pi} \int_{0}^{2 \pi}\{m \cos j t(-q \cos q t \sin m t \sin r t+ \\
& +m \sin q t \cos m t \sin r t+r \sin q t \sin m t \cos r t)+ \\
& +j \cos m t(-q \cos q t \sin r t \sin j t+ \\
& +r \sin q t \cos r t \sin j t+j \sin q t \sin r t \cos j t)\} d t .
\end{aligned}
$$

For $r^{2}=q^{2}+m^{2}+j^{2}$, we have $j+r \neq|m-q|, r+m \neq|j-q|, r+q \neq|m-j|$, $m+j \neq|q-r|$, hence

$$
\begin{aligned}
b_{j m r}^{(q)}+b_{m j r}^{(q)} & =\frac{q^{2} r}{2 \pi} \int_{0}^{2 \pi}\left(m^{2} \sin (q+j) t \sin (r+m) t+\right. \\
& +j^{2} \sin (q+m) t \sin (j+r) t+ \\
& +\frac{1}{2}\left\{(q+r)(m-j)+j^{2}-m^{2}\right\} \sin (m-j) t \sin (q-r) t+ \\
& \left.+\frac{1}{2}\left\{(r-q)(m+j)-m^{2}-j^{2}\right\} \sin (m+j) t \sin (r+q) t\right) d t
\end{aligned}
$$


Then, we might have non zero terms if either $r+m=q+j$, or $j+r=q+m$, or $r+q=m+j$. It is then easy to check that in all these cases the integral vanishes.

Acknowledgements. The author deeply acknowledge John Toland for his careful reading of the manuscript and his interest in a formulation avoiding Fourier series. He showed how to obtain directly (10), (11), improving a previous version of the author in avoiding an originally mysterious change of variable.

\section{References}

[1] C.J.Amick, J.F.Toland. The semi-analytic theory of standing waves. Proc. R. Soc. Lond. A 411, 123-137, 1987.

[2] P.J.Bryant, M.Stiassnie. Different forms for nonlinear standing waves in deep water. J.Fluid Mech. 272, 135-156, 1994.

[3] W.Craig, P.Worfolk. An integrable normal form for water waves in infinite depth. Physica D, 84, 513-531, 1995.

[4] W.Craig. Birkhoff normal forms for water waves. Comptemp. Math. 200, 57-74, 1996.

[5] A.I.Dyachenko, V.E.Zakharov. Is free-surface hydrodynamics an integrable system? Phys. Lett. A, 190, 144-148, 1994.

[6] G.Iooss. Semianalytic theory of standing waves in deep water, for several dominant modes. Proc. Roy. soc. London A, 455, 3513-3526, 1999.

[7] P.I.Plotnikov, J.F.Toland. Nash-Moser theory for standing water waves. Arch. Rat. Mech. Anal. (to appear).

[8] L.N.Schwartz, A.K.Whitney. A semi-analytic solution for nonlinear standing waves in deep water. J.Fluid Mech. 107, 147-171, 1981.

[9] V.E.Zakharov. Stability of periodic waves of finite amplitude on the surface of deep fluid. J.Appl. Mech. Tech. Phys. 2, 190-194, 1968. 